\title{
A role for actin arcs in the leading-edge advance of migrating cells
}

\author{
Dylan T. Burnette ${ }^{1}$, Suliana Manley ${ }^{1}$, Prabuddha Sengupta ${ }^{1}$, Rachid Sougrat $^{1}$, Michael W. Davidson ${ }^{2}$, \\ Bechara Kachar ${ }^{3}$ and Jennifer Lippincott-Schwartz ${ }^{1,4}$
}

Epithelial cell migration requires coordination of two actin modules at the leading edge: one in the lamellipodium and one in the lamella. How the two modules connect mechanistically to regulate directed edge motion is not understood. Using live-cell imaging and photoactivation approaches, we demonstrate that the actin network of the lamellipodium evolves spatio-temporally into the lamella. This occurs during the retraction phase of edge motion, when myosin II redistributes to the lamellipodial actin and condenses it into an actin arc parallel to the edge. The new actin arc moves rearward, slowing down at focal adhesions in the lamella. We propose that net edge extension occurs by nascent focal adhesions advancing the site at which new actin arcs slow down and form the base of the next protrusion event. The actin arc thereby serves as a structural element underlying the temporal and spatial connection between the lamellipodium and the lamella during directed cell motion.

Migrating cells advance by net protrusion at their front leading edge and retraction at their rear ${ }^{1}$. Two regions define the leading edge: the lamellipodium, extending $\sim 3-5 \mu \mathrm{m}$ from the cell edge and consisting mostly of dynamic, criss-crossed actin filaments ${ }^{2-6}$, and the lamella, found immediately behind the lamellipodium and composed of bundled actin filaments in association with focal adhesions ${ }^{5,7,8}$. The lamellipodial actin module extends the cell edge by insertion of actin monomers into filament ends apposed to the leading membrane and their regulated turnover through actin treadmilling ${ }^{9-11}$. The lamellar actin module, on the other hand, assembles a contractile network for traction, consisting of bundled filaments, myosin II and focal adhesions ${ }^{7,8,12}$. Originally, the activities of the lamellipodial and lamellar actin modules were thought to drive cell motion by acting within one integrated system, with myosin II working at a distance from the cell edge $\mathrm{e}^{3,13}$. Later, single-particle tracking of actin speckles using fluorescence speckle microscopy (FSM) indicated that a layer of actin extends from the lamella to the cell edge to control forward cell movement ${ }^{11,14}$. This led to new proposals for cell crawling involving myosin II contractility in the lamella pulling on the back of the lamellipodium ${ }^{15}$. Nevertheless, it is still unclear exactly how lamellipodial and lamellar actin modules interact during cell crawling ${ }^{16-18}$.

One obstacle to investigating how the lamellipodium and lamella actin modules connect mechanistically to mediate cell crawling is that the leading edge is both structurally heterogeneous and highly dynamic $^{5,19}$. Marked changes in actin organization occur as the edge undergoes protrusion and retraction on the timescale of minutes ${ }^{5}$.
As a result of this, previous maps of FSM speckle turnover events averaged over many protrusion/retraction cycles ${ }^{11,17}$ and singlesnapshot electron micrographs of actin distribution ${ }^{6,20}$ may not fully reveal how overall actin structure at the leading edge changes to mediate cell movement. Here, we focus on this issue by examining actin turnover with high temporal and spatial resolution, and by visualizing the overall structural dynamics of the actin cytoskeleton over time. We show that the actin network of the lamellipodium evolves spatio-temporally into the lamella during the retraction phase of edge motion. This evolution is mediated by myosin II, which redistributes to the cell edge at the beginning of the retraction phase of edge motion, condensing the lamellipodial actin into an arc-shaped actin bundle parallel to the edge. A model is presented in which the actin arc serves as the structural element underlying the temporal and spatial connection between the lamellipodium and the lamella to help mediate cell crawling.

\section{RESULTS}

\section{Actin-filament organization at the leading edge}

Examination of rotary-shadowed cells by electron microscopy ${ }^{4,21}$ reveals a criss-crossed actin network in the lamellipodium adjacent to the plasma membrane and a bundled actin network in the lamella farther away from the edge $(\sim 2-5 \mu \mathrm{m}$; Fig. 1a), similar to previous reports ${ }^{3,5,6}$. No lamellipodial sheet raised above the lamella, proposed in previous studies ${ }^{15}$, is observed.

Fluorescence speckle microscopy (FSM) using actin tagged to the photoconvertable protein, tdEos, which converts from green

\footnotetext{
${ }^{1}$ National Institute of Child Health and Human Development, National Institutes of Health, Bethesda, Maryland 20892, USA. ${ }^{2}$ National High Magnetic Field Laboratory and Department of Biological Science, Florida State University, Tallahassee, Florida 32310, USA. ${ }^{3}$ National Institute on Deafness and Other Communication Disorders, National Institutes of Health, Bethesda, Maryland 20892, USA.

${ }^{4}$ Correspondence should be addressed to J.L-S. (e-mail: lippincj@mail.nih.gov)
}

Received 17 August 2010; accepted 6 January 2011; published online 20 March 2011; DOI: 10.1038/ncb2205 
a

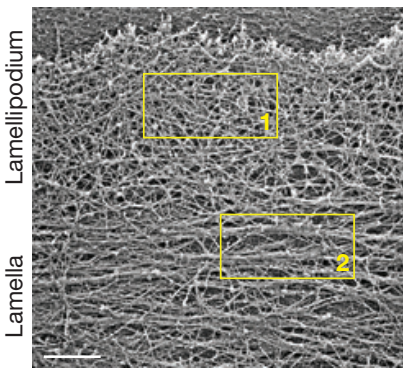

C

Rearward-speckle-flow kymograph
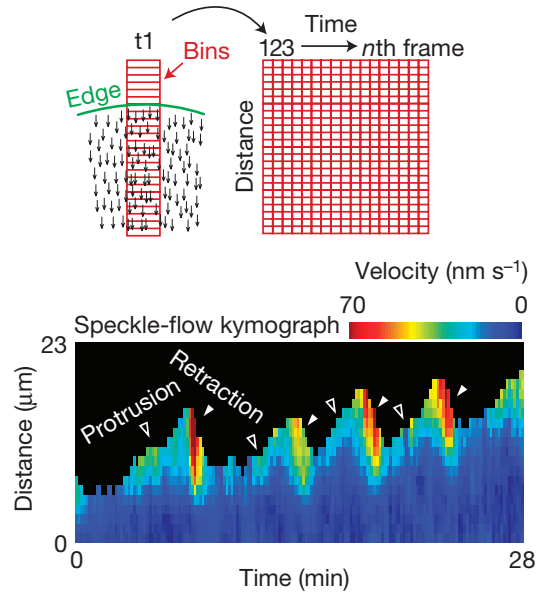

e
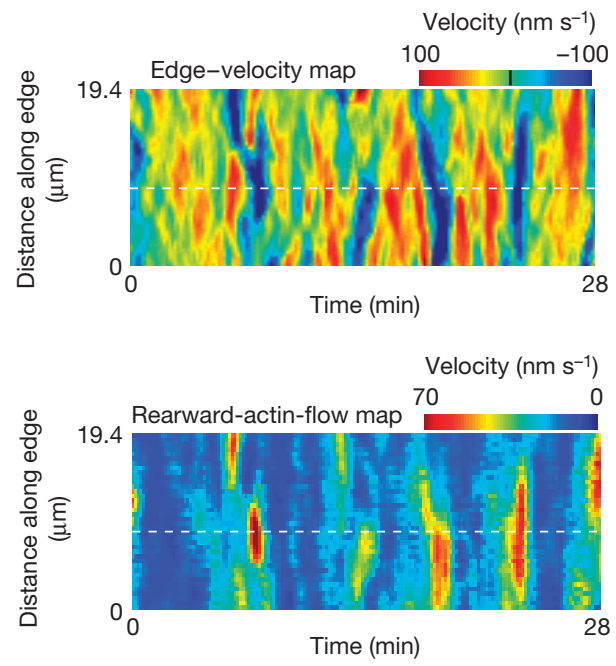

Figure 1 Retrograde-actin-flow rates change several times over a single edge-protrusion/retraction cycle. (a) Electron micrograph of a rotary-shadowed cell after live-cell extraction. Areas 1 and 2 show actin-filament organization in the lamellipodium and lamella, respectively. Scale bar, $1 \mu \mathrm{m}$. (b) Actin-tdEos speckle image of an entire PtK1 cell with a corresponding FSM flow map and a higher magnification of the leading-edge flow. LM, lamella; LP, lamellipodium. Vector colours reflect flow speed (colour bar), and arrows reflect direction. Scale bar, $10 \mu \mathrm{m}$. (c) Edge-motion rates relative to retrograde actin flow. Schematic of how the speckle-flow data were binned, and the resulting rearward-speckle-flow kymograph showing the change in retrograde-flow rates during protrusion (open arrows) and retraction (filled arrows) of

to red emission, permitted examination of actin-flow pattern at high temporal resolution ( $5 \mathrm{~s}$ intervals) over long periods of time (25-60 min; Supplementary Movie S1). By replenishing fluorescently

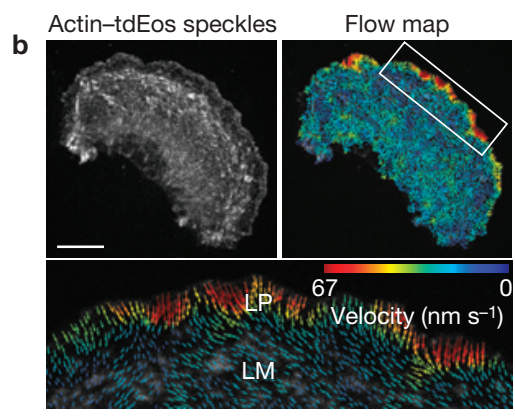

d

Edge-protrusion/retraction map

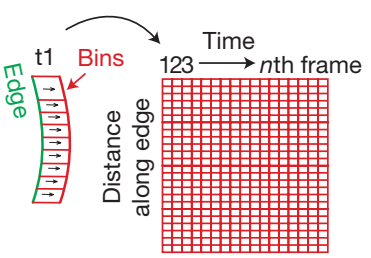

Rearward actin flow behind edge map

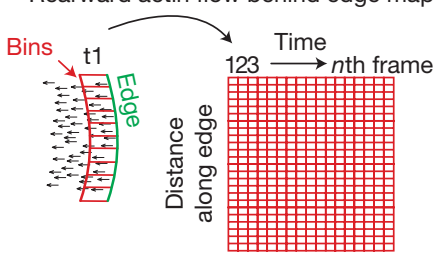

f
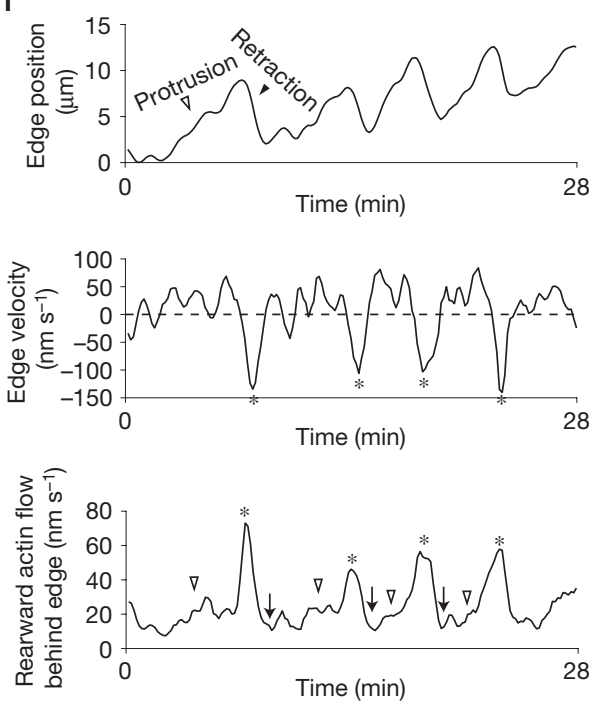

the leading edge. Each data bin was $5 \mu \mathrm{m}$ across and $1 \mu \mathrm{m}$ high. (d) Schematics of how the edge-protrusion and retrograde-flow data were binned across the leading edge. Bins for edge protrusion were set at $500 \mathrm{~nm}$ parallel to the edge. Bins for retrograde flow were set at $1 \mu \mathrm{m}$ parallel to the edge and $3 \mu \mathrm{m}$ into the cell. (e) Edge-protrusion/retraction velocity and rearward actin velocity maps of the same cell used for the kymograph in c. (f) Edge position, edge velocity and rearward actin flow of the region denoted by the dashed lines in e plotted over time. Asterisks in edge-velocity and rearward-actin-flow graphs denote retractions and arrowheads denote protrusions corresponding to increases in rearward actin flow. Arrowheads denote slowing rearward actin flow immediately after edge retraction.

tagged actin monomers through photoactivation during imaging, we achieved longer time-lapse recordings than most previous actin speckling studies. This allowed us to compare actin-retrograde-flow 
rates during both protrusion and retraction phases of edge motion. An overall fast-flow zone of actin filaments in the lamellipodium and a slow-flow zone in the lamella are seen when the data are averaged over many protrusion/retraction cycles, as previously reported ${ }^{11,22}$ (Fig. 1b). However, when the data are analysed in kymograph form (Fig. 1c, diagram) to look for changes in rearward-actin-flow velocities over time, a large increase in flow velocity is seen during edge retraction (Fig. 1c, filled arrowheads), compared with during protrusion (open arrowheads). In the cell analysed in Fig. 1c, in which the edge undergoes four full protrusion/retraction cycles during 28 min of imaging, flow rates increase during each retraction event. Graphs coloured-coded for speed (Fig. 1d,e) also reveal that the retraction phase of edge motion is always accompanied by a significant increase in the rate of rearward actin-filament flow (Fig. 1f, asterisks), with a smaller increase occurring during edge protrusion (Fig. 1f, open arrowheads). Thus, it is possible that a major change in the lamellipodial actin module occurs when the leading edge switches from protruding to retracting, which correlates with rapid actin-filament movement rearward.

\section{Actin-turnover kinetics at the leading edge}

Next, we examined lamellipodial actin-filament turnover rates during edge protrusion/retraction. The necessary temporal resolution was achieved by photoconverting a subpopulation of actin-tdEos in the lamellipodium as it underwent either protrusion or retraction (Fig. 2a-d). As this subpopulation was now spatially highlighted, we could specifically track the fate and lifetime of these actin filaments.

During edge protrusion, photoconverted actin-tdEos molecules completely turn over within 1-2 min, with few, if any, actin filaments transferring to the lamella (Fig. 2a,c). This turnover presumably occurs through filament depolymerization. In contrast, during edge retraction, a subset of photoconverted actin-tdEos molecules persists within actin filaments (Fig. 2b, yellow arrowheads and Fig. 2c,d). These compact into an arc-shaped bundle that moves rearward into the lamellar region. The new actin arc then joins the non-photoconverted transverse actin-filament bundles below it (Fig. 2d). The half-life of actin filaments in the lamellipodium during retraction resembles that of the actin filaments in the lamella (Fig. 2e,f). Thus, as the edge switches from protruding to retracting, the lamellipodial actin converts into an actin arc, whose lifetime and structure are similar to those of actin arcs in the lamella.

\section{Characterization of actin-arc formation and behaviour}

Time-lapse imaging of monomeric red fluorescent protein (mRFP)tagged actin in live cells allowed us to monitor the behaviour of actin arcs in relation to other actin structures at the leading edge (Fig. $3 \mathrm{a}-\mathrm{c}$ ). These included the actin-filament networks in the lamellipodium (Fig. 3a) and the perpendicular actin filaments associated with focal adhesions $^{12}$ (Fig. 3a, yellow arrowheads). A new actin arc forms in the lamellipodium during every edge retraction (Fig. 3a-c, red arrowheads and arrows and Supplementary Movie S2). The new actin arc moves rearward to become part of the existing actin-arc population in the lamella during the next protrusion phase (Fig. 3a,b, red arrowheads). Time-lapse imaging of cells co-expressing the focal-adhesion protein zyxin and actin revealed that actin-arc bundles can form before their co-localization with focal adhesions (Fig. 3d).
The velocity of an actin arc decreases when it comes in contact with focal adhesions (Fig. 3d, arrowheads), possibly owing to coupling between actin filaments and focal-adhesion proteins ${ }^{23,24}$. Consistent with this, rearward motion of regions of an actin arc nearest to focal adhesions is slower than that of regions furthest from focal adhesions. This creates a bow-like appearance in the arc as it enters the lamella (Fig. 3a, red arrowheads). In rare cases, a small actin bundle is left behind by the protruding lamellipodium (Supplementary Fig. S1). This bundle stays relatively stationary until it merges with the retracting primary actin arc (Supplementary Fig. S1). In the lamella, new actin-arc arrival at the front of the arc stack is balanced by actin-arc loss and disassembly at the rear (Fig. 3b,c, red and yellow arrowheads). This results in treadmilling of arcs through the stack, with arcs periodically dissociating from the back of the lamella and disappearing (Fig. 3b, yellow arrowheads and Supplementary Movie S2).

Velocity profiles of the edge over time were analysed in protrusion/retraction maps (colour-coded for velocity), as well as in graphs that showed single points along the edge (Fig. 3e,f). This reveals that the frequency of protrusion and retraction of the cell edge (and hence frequency of arc formation) remains constant over time. Fourier analysis (used to determine the variation in the velocity with time at each point along the edge of an individual cell) shows a primary frequency of fluctuation of edge velocity (Fig. 3g). The entire leading edge of a cell thus exhibits the same protrusion/retraction frequency. Analysis of the protrusion/retraction period reveals that each cell has a characteristic protrusion/retraction period for edge motion (and hence arc-formation rate), with the value varying considerably from cell to cell (Fig. $3 \mathrm{~h}$ ).

\section{Role of myosin II in actin-arc formation}

Mature actin arcs in the lamella are myosin II-containing contractile bundles and inhibition of myosin II activity decreases the number of arcs in both the central domain of neuronal growth cones and the lamella of epithelial cells ${ }^{12,25}$. To investigate the role of myosin II in arc formation, cells co-expressing green fluorescent protein (GFP)-tagged myosin IIA and actin-mRFP were monitored by time-lapse imaging. During edge protrusion, myosin II is associated with actin arcs of the lamella with virtually none found in the lamellipodium (Fig. 4a). This finding supports previous work indicating that myosin II is restricted to the lamella and is absent from the lamellipodium ${ }^{8,13,26}$. When myosin II was monitored over a protrusion/retraction cycle, however, it appears in the lamellipodium at the peak of protrusion (Fig. $4 \mathrm{~b}$ and Supplementary Movie S3). Myosin II then translocates backward, mirroring the position and movement of the retracting actin arc (Fig. 4c, white arrows). Myosin II also appears on the smaller actin arcs that are occasionally left behind by the protruding lamellipodium (Fig. 4c, yellow arrowheads). This myosin II population remains stationary and then moves rearward with the primary actin arc during edge retraction (Fig. 4c, yellow arrowheads). Myosin II thus localizes to actin arcs formed in the lamellipodium during edge retraction.

To test whether myosin II is involved in arc formation, we imaged cells treated with blebbistatin, a specific inhibitor of myosin II ATPase activity (Fig. 4d). After treatment, no actin arcs form and stacked arcs in the lamella disappear. Indeed, the boundary between lamellipodial and lamellar actin modules is lost in treated cells. This indicates that myosin II activity is required for arcs to form and be maintained, both in the lamellipodium and lamella. Interestingly, before retracting, 
a

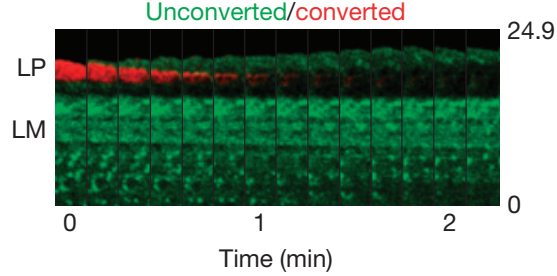

C

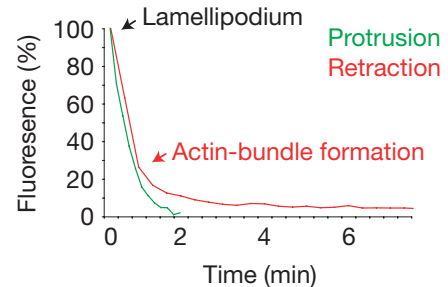

b
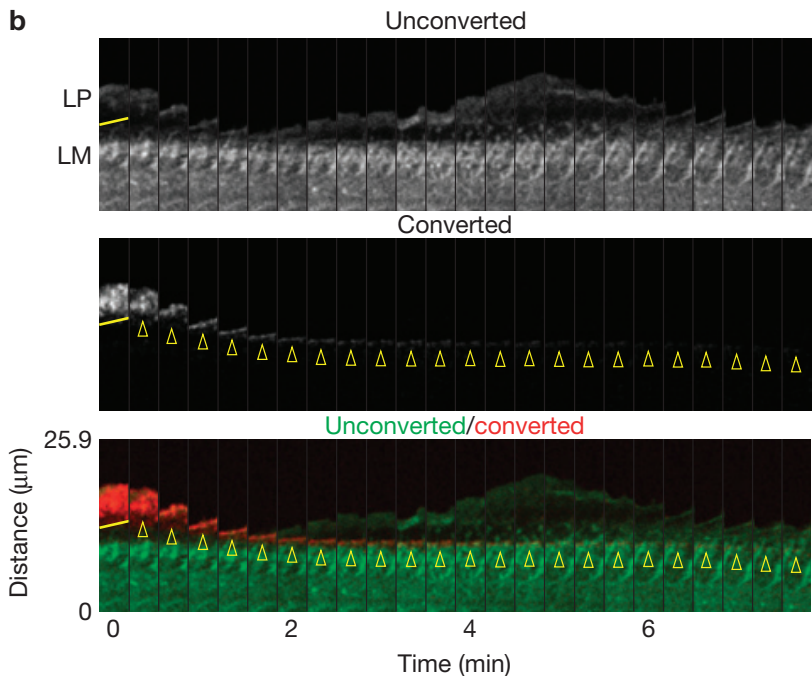

d

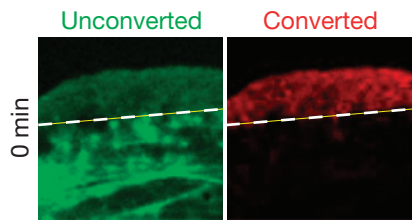

e

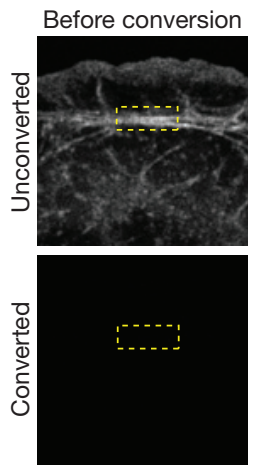
$0 \min$

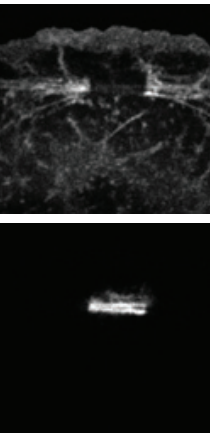

$6 \min$

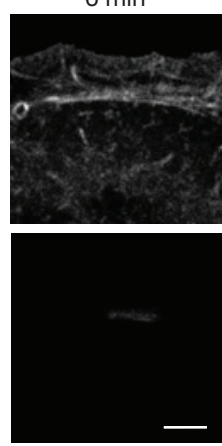

Figure 2 Differential actin-filament turnover during protrusion and retraction. (a) Montage of unconverted actin-tdEos (green) at the edge and actin-tdEos photoconverted (red) in the lamellipodium during edge protrusion. LM, lamella; LP, lamellipodium. (b) Montage of unconverted and converted actin-tdEos molecules during edge traction. Yellow line denotes region of photoconversion and arrowheads denote actin-bundle formation. (c) Quantification of fluorescence loss of converted actin-tdEos molecules in the lamellipodium during edge

the edge protrudes out farther in the presence of blebbistatin when compared with the control. This indicates that myosin II-based actin-arc formation regulates, but is not the sole controlling element in, the switch between edge protrusion and retraction.

\section{Relating edge protrusion/retraction to cell crawling}

Leading-edge velocity and position data in crawling cells permitted a comparison of the amplitude/frequency of edge protrusion/retraction and cell-crawling rate (Fig. 5a,b). This allowed us to determine whether the frequency of the edge's protrusion/retraction cycle is related to how fast the edge exhibits net extension ${ }^{13}$. An inverse relationship exists between the amplitude and frequency of edge protrusions
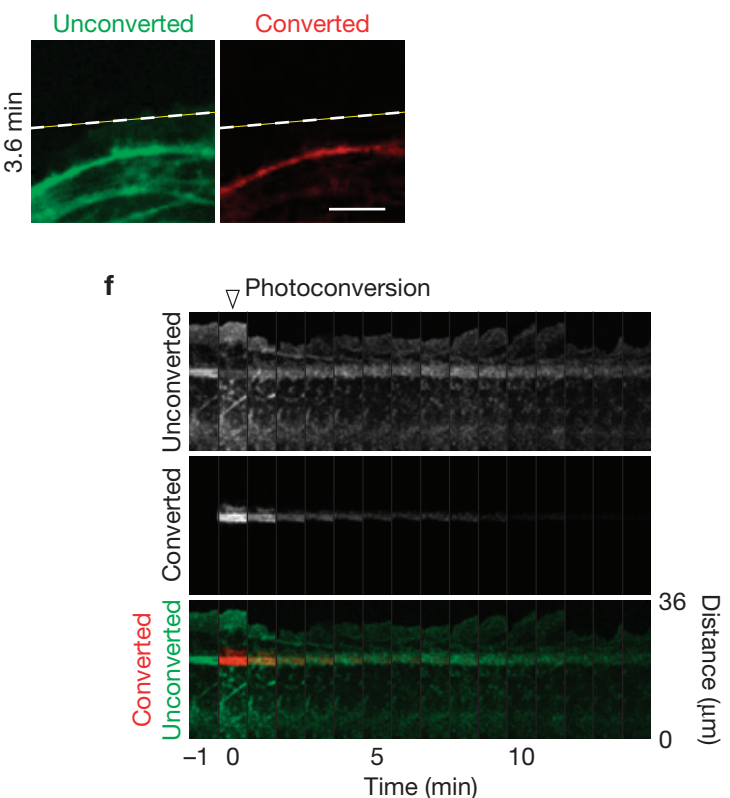

protrusion (green line) or retraction (red line). (d) Still frames showing that the actin bundle formed after retraction is arc shaped. Scale bar, $5 \mu \mathrm{m}$. (e) Montage showing unconverted and converted channels before, $0 \mathrm{~min}$, and $6 \mathrm{~min}$ after photoconversion of actin-tdEos molecules incorporated into actin arcs in the lamella (dashed outline). Scale bar, $10 \mu \mathrm{m}$. (f) Time montage showing the recovery of fluorescence from the unconverted channel and loss of fluorescence from the converted channel of actin-tdEos in the lamella.

(Fig. 5c). However, we found no correlation between the migration rate of a cell and either the amplitude, or the frequency, of its protrusions (Fig. 5d,e). Indeed, both the frequency and amplitude of protrusion in an individual cell remain constant whether or not the cell is crawling. Instead, the main, and obvious, difference in edge motion between fast- and slow-moving cells is the ratio between the amplitude of protrusion and amplitude of retraction (Fig. 5f). Cells exhibiting net movement have retraction amplitudes that are smaller than protrusion amplitudes (Fig. 5b), whereas those that do not move forward efficiently have amplitudes of protrusion and retraction that are roughly the same. This indicates that differences in actin dynamics per se do not explain differences in migration rates. Consistent with this, 
a

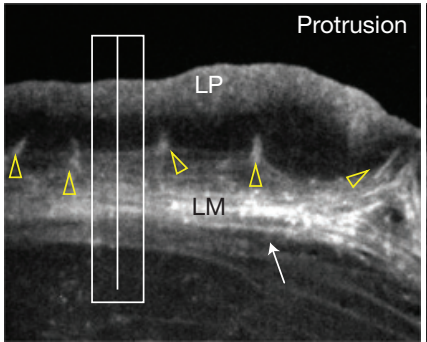

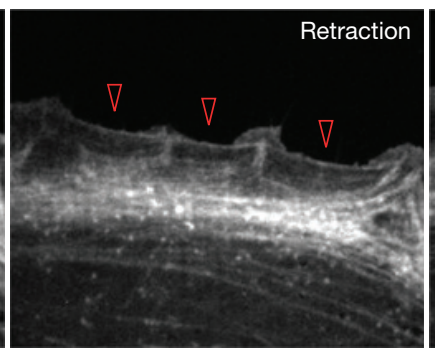
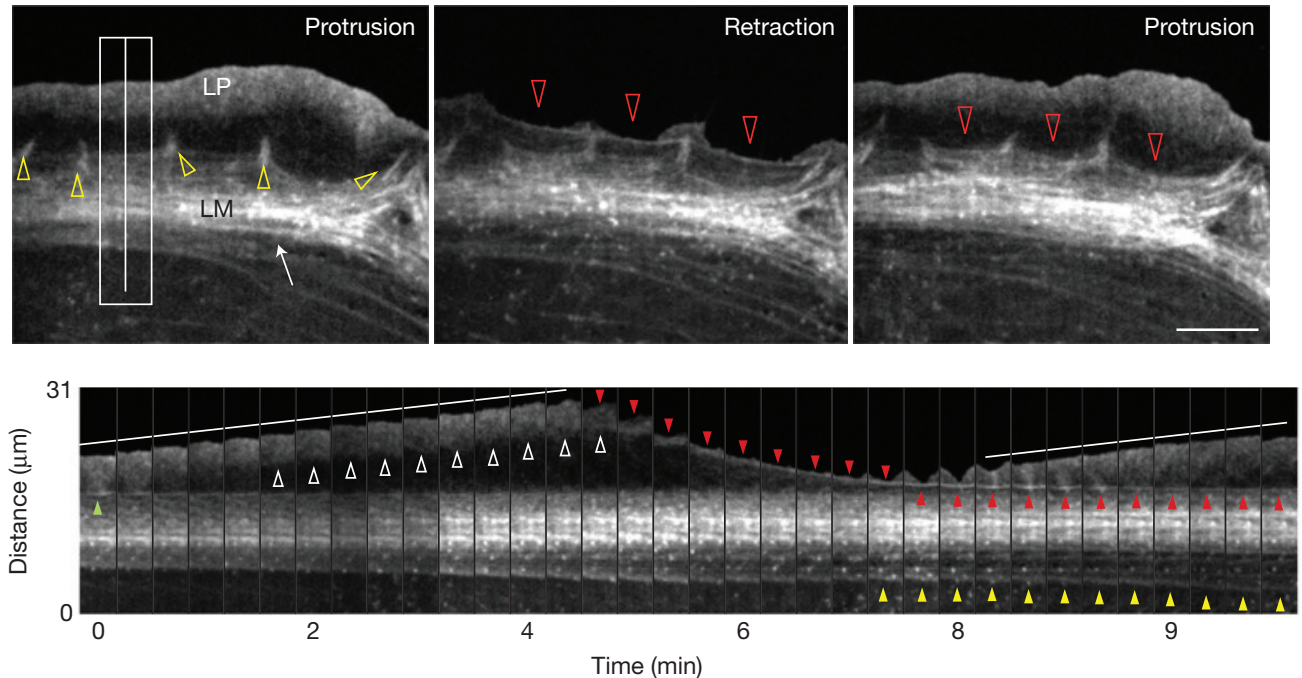

c

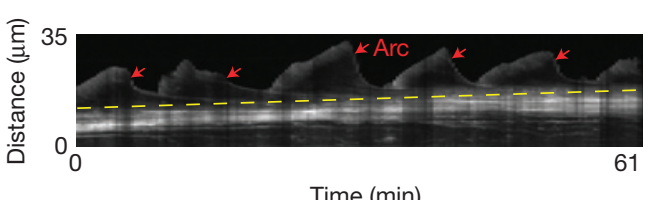

e

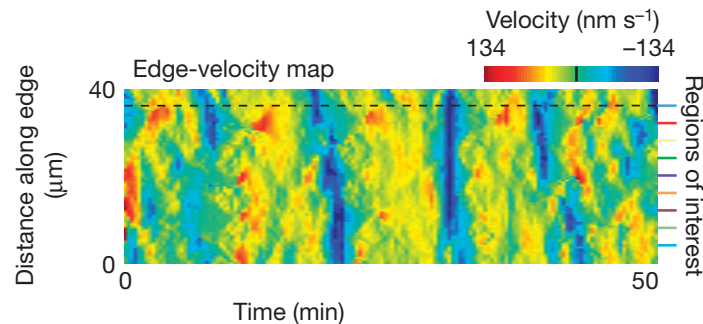

f

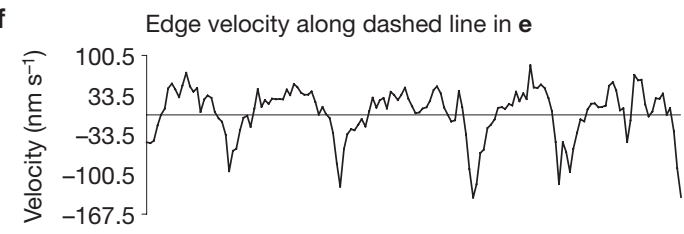

d

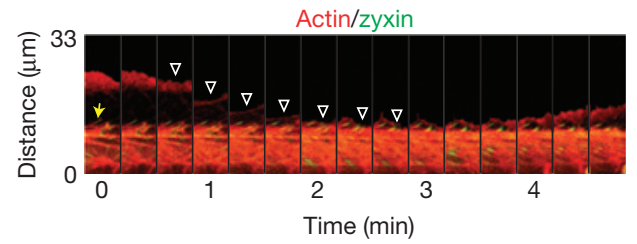

$\mathbf{9}$

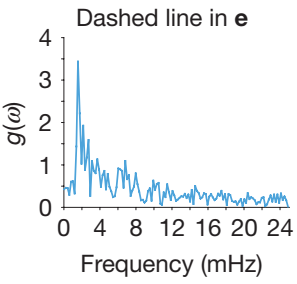

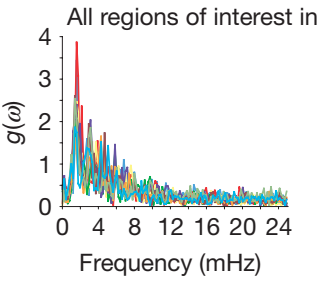

Frequency $(\mathrm{mHz})$

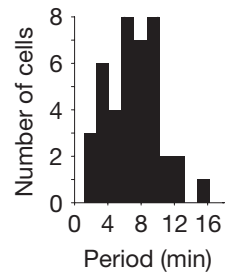

Figure 3 Actin-arc dynamics at the leading edge. (a) Three frames of a time-lapse recording of actin-mRFP showing the cytoskeletal organization at the leading edge during the transition from protrusion to retraction and back to protrusion. LP denotes actin network in the lamellipodium and arrow denotes actin arcs in the lamella (LM). Yellow arrowheads show actin filaments associated with focal adhesions. Red arrowheads show a newly forming actin arc. Scale bar, $10 \mu \mathrm{m}$. (b) Time-lapse montage of the area outlined in a showing the formation of an actin arc (red arrowheads) between protrusion events (white lines). Green arrowhead shows the actin arc formed during the previous retraction event. White arrowheads show zone of actin depolymerization during edge protrusion. Yellow arrowheads show the removal of actin arcs. The change in intensity from frame 10 to

crawling and non-crawling cells exhibit similar patterns of rearward actin flow (Fig. 5g,h).

\section{Defining the protrusion base in crawling cells}

The placement of new focal adhesions in front of old adhesions is a hallmark of crawling cells and seems to advance the boundary

11 is due to focusing. (c) Kymograph of the line in a showing multiple protrusion and retraction events over $1 \mathrm{~h}$. Red arrows denote the first frame an actin arc was observable and yellow dashed line shows lamellar advance. (d) Zyxin-mCherry and monomeric GFP (mGFP)-tagged actin montage showing that an actin arc can form (white arrowheads) before coming in contact with focal adhesions (yellow arrow). Actin was psuedo-coloured red for consistency with other figures and zyxin was psuedo-coloured green. (e) Protrusion/retraction map showing edge-velocity (shown by colour bar) changes over time across the edge of the cell in a. (f) Plot of the edge velocity from the dashed line in e. (g) Fourier transforms of the velocity profiles represented by the coloured lines in e. (h) Distribution of the period of the protrusion/retraction cycle among 41 cells.

between the lamellipodium and lamella ${ }^{27,28}$. Recent work has also indicated that whole adhesions can move away from the leading edge $^{29}$. To investigate this further, we examined the dynamics of focal adhesions in relation to net edge motion. Figure 6a shows focal adhesions labelled with zyxin-mCherry before (purple) and after (green) imaging for $14 \mathrm{~min}$. Note that several adhesions move 
a

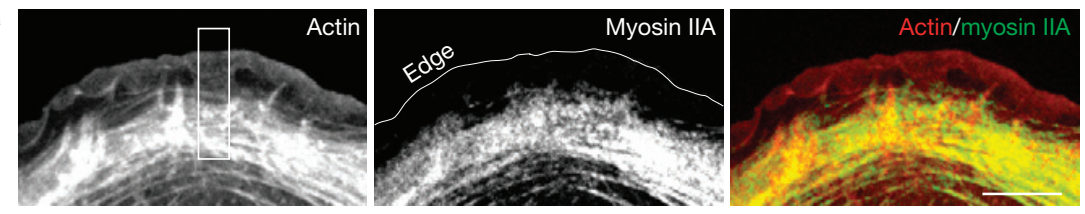

b
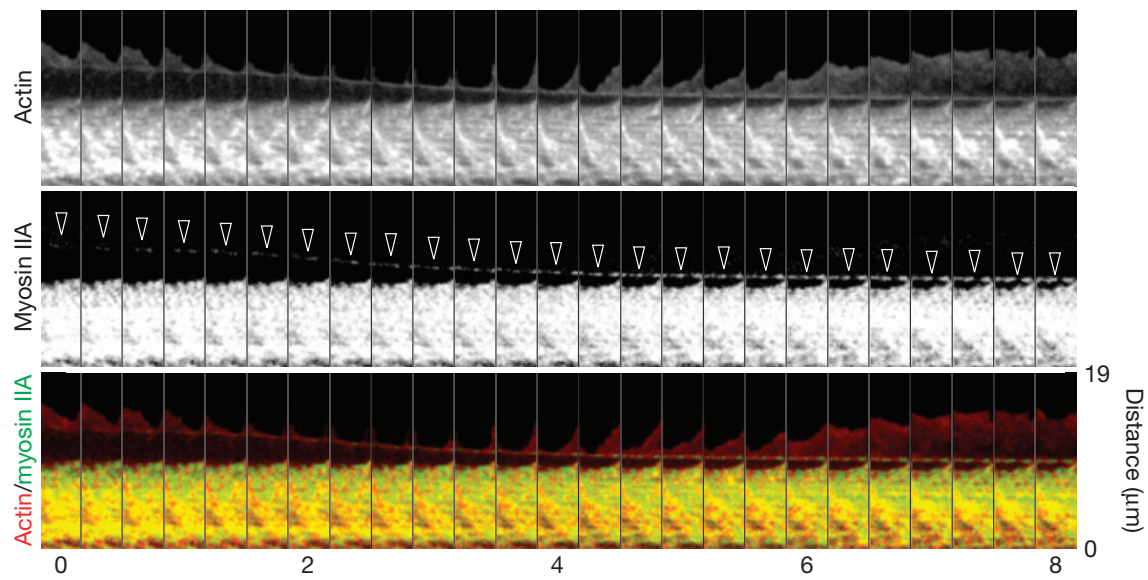
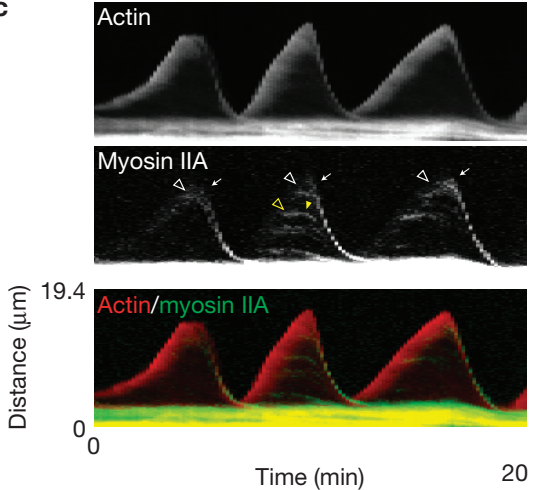

d

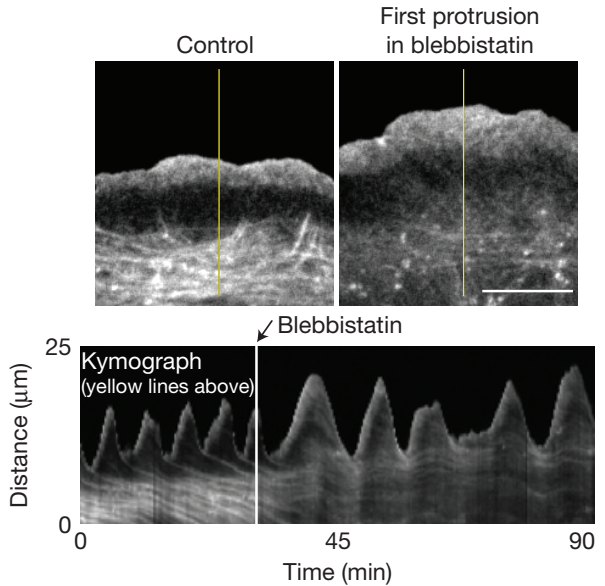

Edge-velocity map

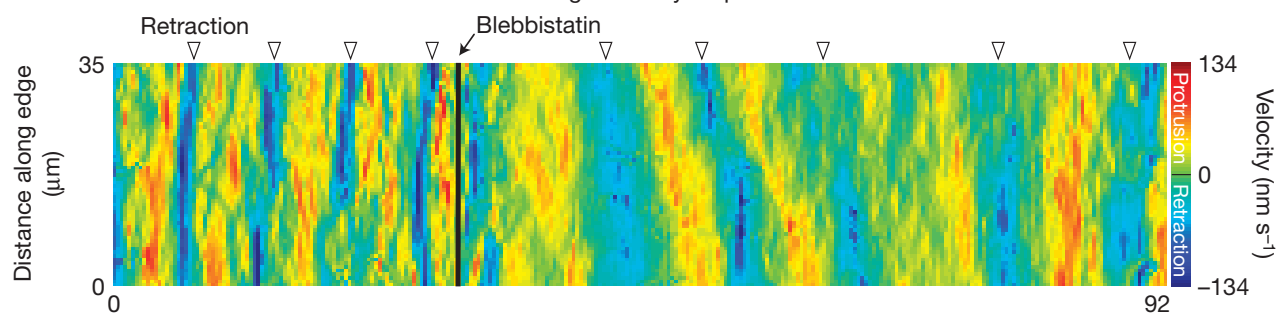

Time (min)

Figure 4 Myosin II activity condenses the lamellipodium into an actin arc. (a) Organization of actin-mRFP, myosin IIA-GFP and overlay during edge protrusion. Myosin II localizes with older actin arcs in the lamella. Scale bar, $10 \mu \mathrm{m}$. (b) Time-lapse montages of actin-mRFP, myosin IIA-GFP and an overlay from the rectangle in a showing the co-localization of myosin II with newly forming actin arcs. Arrowheads show co-translocation of myosin IIA and the newly formed actin arc. (c) Kymograph showing myosin IIA dynamics over three protrusion/retraction cycles. White arrowheads denote the appearance and white arrows denote the movement of myosin

away from the edge over this time period (Fig. 6a, arrows). Tracking adhesions labelled with either zyxin or vinculin over time revealed that less rearward movement of focal adhesions occurs in rapidly crawling
IIA. Yellow arrowhead denotes the appearance and yellow arrow denotes the movement of myosin IIA associated with a small actin bundle left behind by the protruding lamellipodium. (d) Actin-mRFP before and after treatment with $25 \mu \mathrm{m}$ blebbistatin. Kymograph shows the protrusion retraction cycle of the edge before and after blebbistatin treatment. The structure and movement of the actin arcs are diminished in the presence of blebbistatin. (e) Protrusion/retraction map showing edge motion before and after blebbistatin addition (arrow). Edge retractions denoted by arrowheads.

cells and more movement occurs in slow cells (Fig. 6b-d). Similar rearward focal-adhesion movement behaviour is observed with paxillin (Supplementary Fig. S3). 


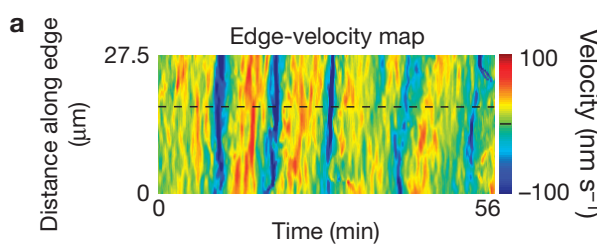

Edge velocity along dashed line above

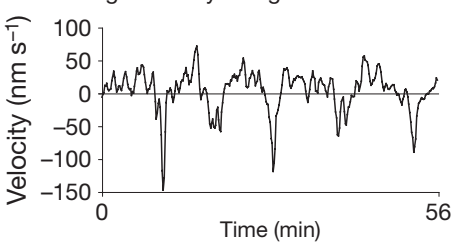

e

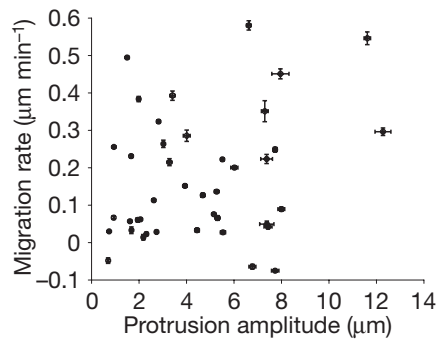

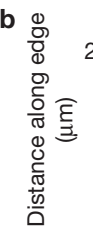

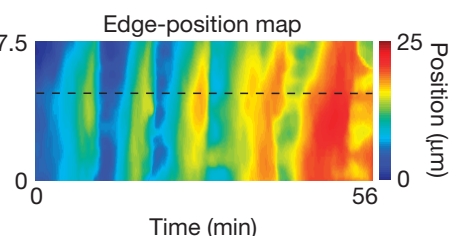

Edge position along dashed line above
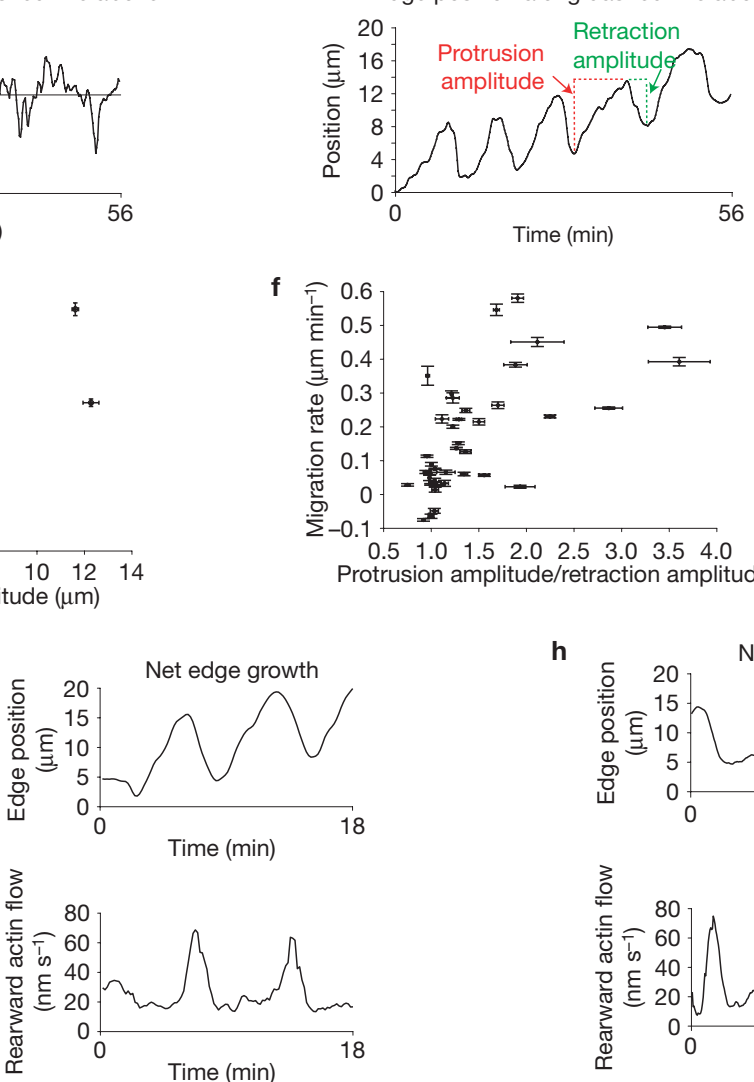

Figure 5 Oscillatory edge motion and net edge extension. (a) Edge-velocity map along the edge and a single region (bottom graph) along the edge from a crawling cell. (b) Edge-position map of the same cell as in a. Edge-position map was created by colour-coding the lowest edge position as blue and the highest as red as in the colour bar. This allows for relative edge position along the same regions as in the velocity map in a to be graphically shown over time. Bottom graph in $\mathbf{b}$ shows the relative edge position at one point along the edge. Red dotted line and green dotted line graphically show the protrusion amplitude and retraction amplitude of one protrusion, respectively. (c) Protrusion amplitudes plotted against edge oscillation frequencies for individual cells. Correlation coefficient: -0.5129 (confidence interval: $-0.7087,-0.2437$ ). (d) Migration rate

Rearward movement of focal adhesions occurs coincident with edge retraction (Supplementary Fig. S2). As actin arcs are forming and moving rapidly during edge retraction and also slow down at focal adhesions (Fig. 3d), we investigated whether new actin arcs interact with newly formed focal adhesions. The white asterisk in the actin montage of Fig. 6e shows the base of the first retraction/protrusion cycle in a fast-crawling cell. This position correlates with where a newly formed actin arc slows down at a pre-existing adhesion (white arrowheads and white asterisk) and a subsequent new protrusion event starts. During this protrusion event, a new focal adhesion is formed (yellow arrowheads). The newly formed actin arc slows at the new adhesion and this position becomes the base of the next protrusion
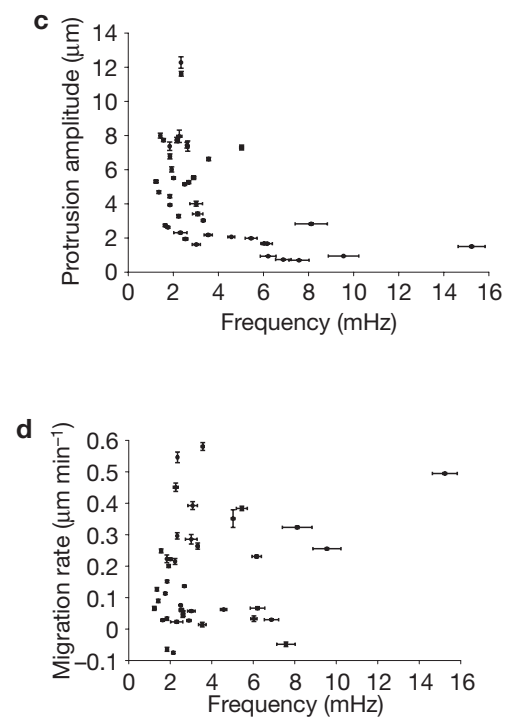

h
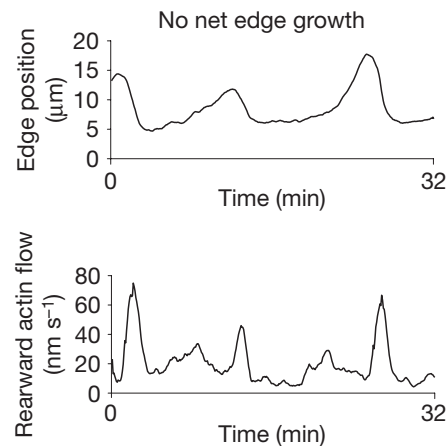

plotted against edge oscillation frequencies for individual cells. Correlation coefficient: 0.1966 (confidence interval: $-0.1182,0.4755)$. (e) Migration rate plotted against protrusion amplitudes for individual cells. Correlation coefficient: 0.2650 (confidence interval: $-0.0464,0.5295)$. (f) Migration rate plotted against the ratio of protrusion and retraction amplitudes in individual cells. Correlation coefficient: 0.4254 (confidence interval: $0.1355,0.6482$ ). For $\mathbf{c}-\mathbf{f}, n=41$ cells. (g,h) Edge position and rearward-flow velocity plotted as in Fig. $1 \mathrm{f}$ for a cell that demonstrates net edge growth (g) and a cell that does not (h). Note the pattern of rearward actin flow is similar in both cells. Pearson's correlation coefficient was used to quantify the correlation and the $95 \%$ confidence interval for each pair was computed using the Fisher transformation.

(Fig. 6e, yellow asterisk). This base is positioned distally, compared with the first protrusion (compare white and yellow asterisks). These results indicate that new-focal-adhesion formation helps advance the cell by slowing down new actin arcs distally to where previous actin arcs are slowed.

In cells that have little net edge extension, new actin arcs move with new adhesions rearward before slowing down together (Fig. 6f, left yellow and right green lines). The same adhesion can move back farther after association with the actin arc from the next retraction event (Fig. 6f, middle yellow line and Fig. 6c,d). The net result is that the cell in Fig. $6 \mathrm{f}$ advances the base of its protrusion retraction cycle little when compared with the cell in Fig. 6e (brackets). Analysis of actin 
a
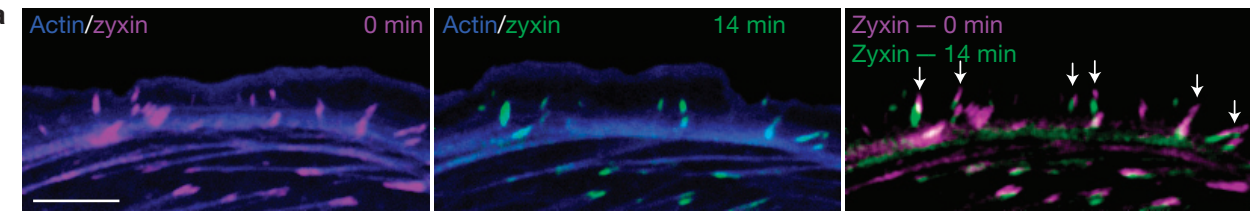

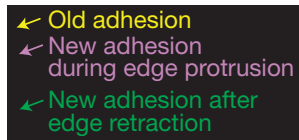

Fast-growth cell in e Adhesion

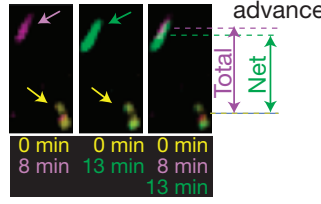

Slow-growth cell in $\mathbf{f}$
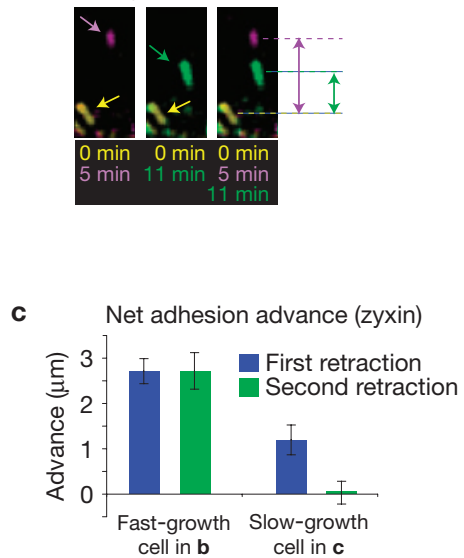

d

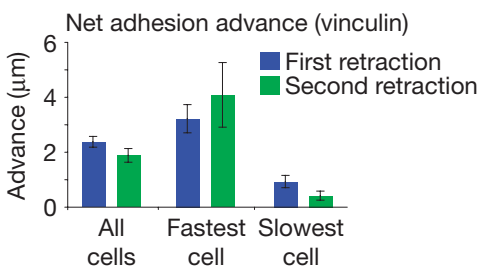

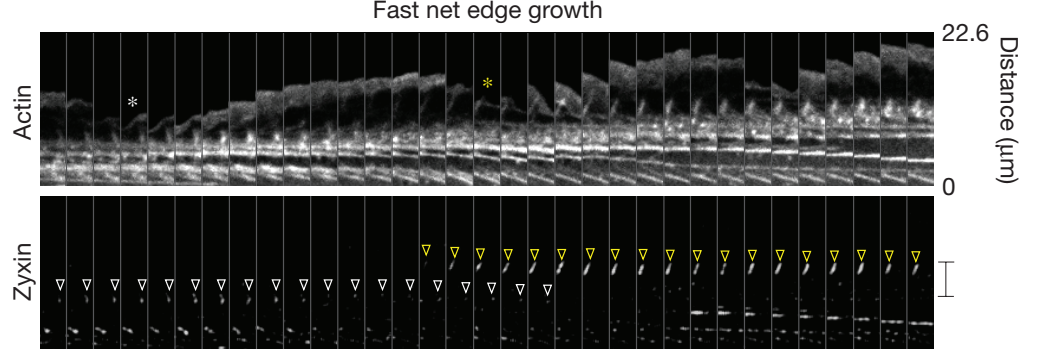

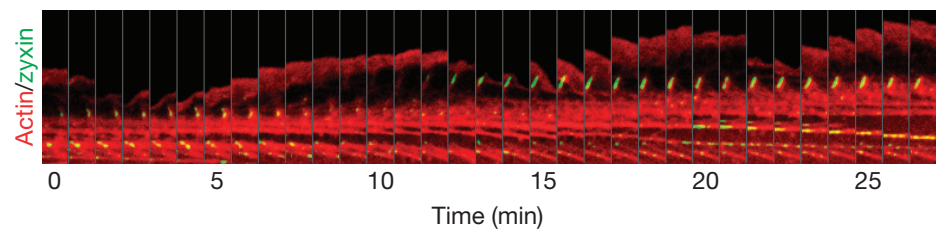

f
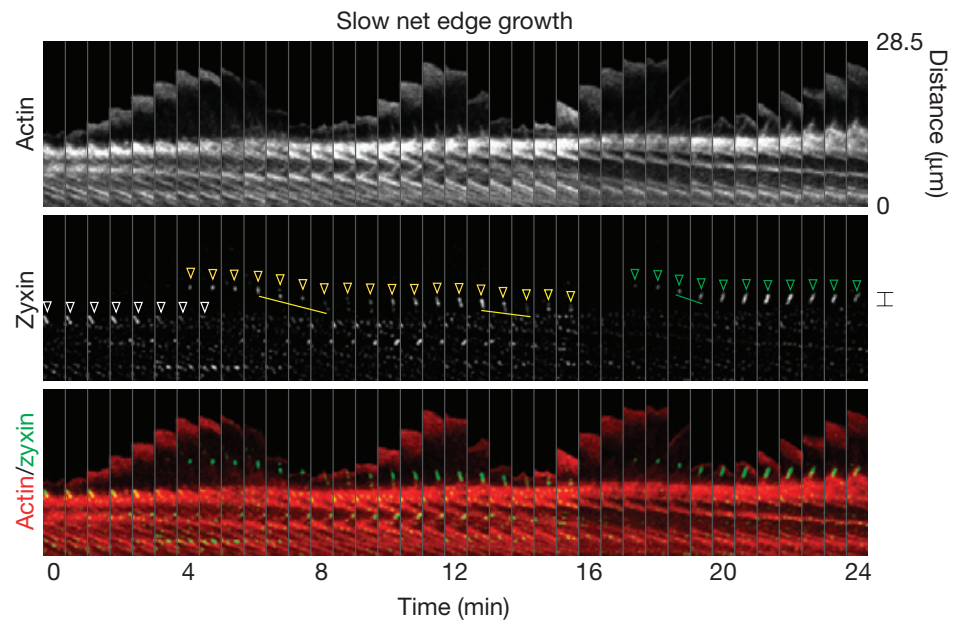

g

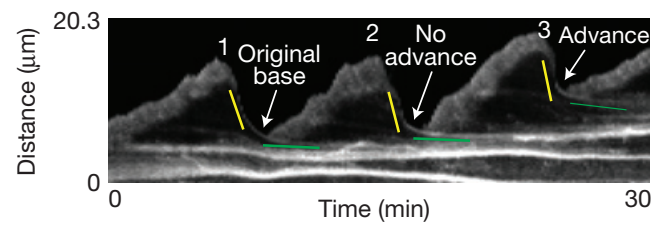

Figure 6 Differential slippage of focal adhesions in crawling versus non-crawling cells correlates with new-actin-arc movement. (a) Overlay of actin-mGFP (blue) and zyxin-mCherry at 0 min (purple) and 14 min (green). Arrows show focal adhesions that move during this time period. Scale bar, $10 \mu \mathrm{m}$. (b) Focal-adhesion movement, compared with pre-existing adhesions. New focal adhesion during protrusion (purple) and after edge retraction (green) in cells with different migrating rates (fast cell- $0.25 \mu \mathrm{m} \mathrm{min}^{-1}$; slow cell- $0.04 \mu \mathrm{m} \mathrm{min}^{-1}$ ). A pre-existing adhesion in each cell is shown in yellow. Total distance of the new adhesion from the previous adhesion during protrusion (purple double-headed arrows) and the net distance after new adhesion slippage (green double-headed arrows) are shown. (c) Net distance between new adhesions and pre-existing adhesions for the fast cell in $\mathbf{b}$ ( $n=38$ adhesions) and a slow cell in $\mathbf{b}$ ( $n=29$ adhesions). Distance was calculated for each new adhesion after the first and second edge-retraction event for which they are associated. (d) Net adhesion advance from focal adhesions labelled with vinculin. Average distance of a population of eight cells ( $n=132$ adhesions), and for the fastest $\left(0.31 \mu \mathrm{m} \mathrm{min}^{-1} ; n=17\right.$ adhesions) and slowest $\left(0.01 \mu \mathrm{m} \mathrm{min}^{-1} ; n=39\right.$ adhesions) cell in the population are shown. Error bars in c and d, s.e.m. (e) Time-lapse montage of actin-mGFP and zyxin-mCherry in a crawling cell. First retraction-to-protrusion transition point (white asterisk) and second transition point (yellow asterisk) are denoted. White arrowheads show the pre-existing focal adhesion. Yellow arrowhead shows a nascent adhesion appearing and maturing (growing larger). (f) Similar time-lapse montage as in $\mathbf{e}$ in a non-crawling cell. Pre-existing adhesion (white arrowheads) and new focal adhesions (yellow and green arrowheads) are shown. Adhesions move rearward (lines) during edge retraction. Brackets in e,f show the net movement of the base of the protrusion-retraction cycle/focal-adhesion advance. (g) Kymograph from a cell that increases its rate of migration. There is no advance of the base protrusion after two protrusion/retraction cycles (arrows), but there is advance after the third cycle (arrow). Yellow and green lines denote rapid and slow actin-arc translocation, respectively. 
a

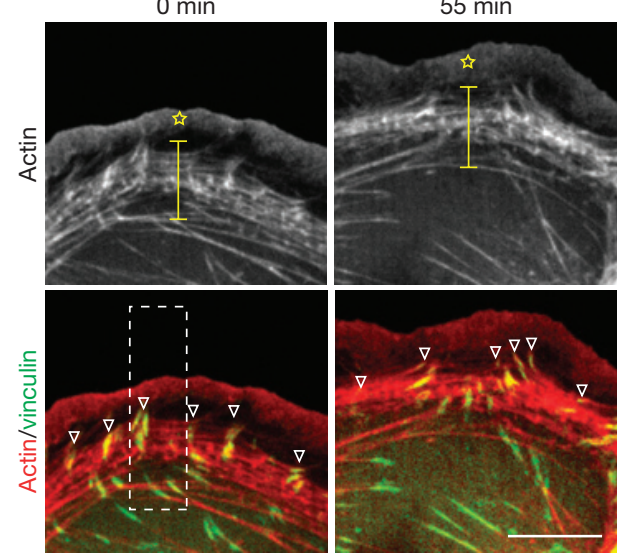

b
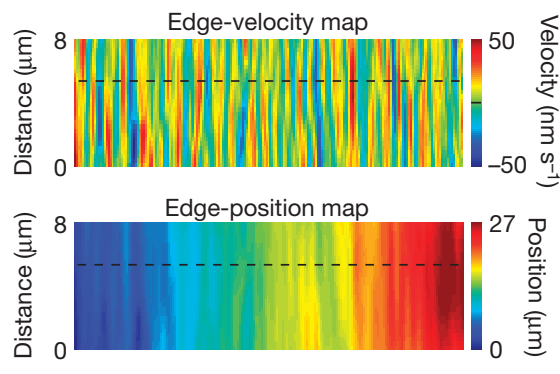

Kymograph

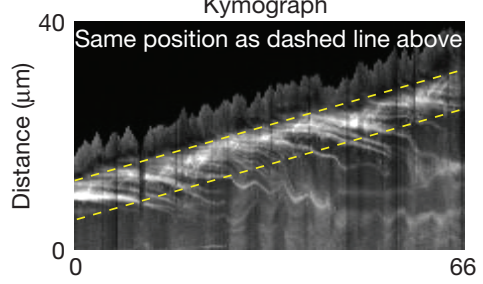

Time (min)
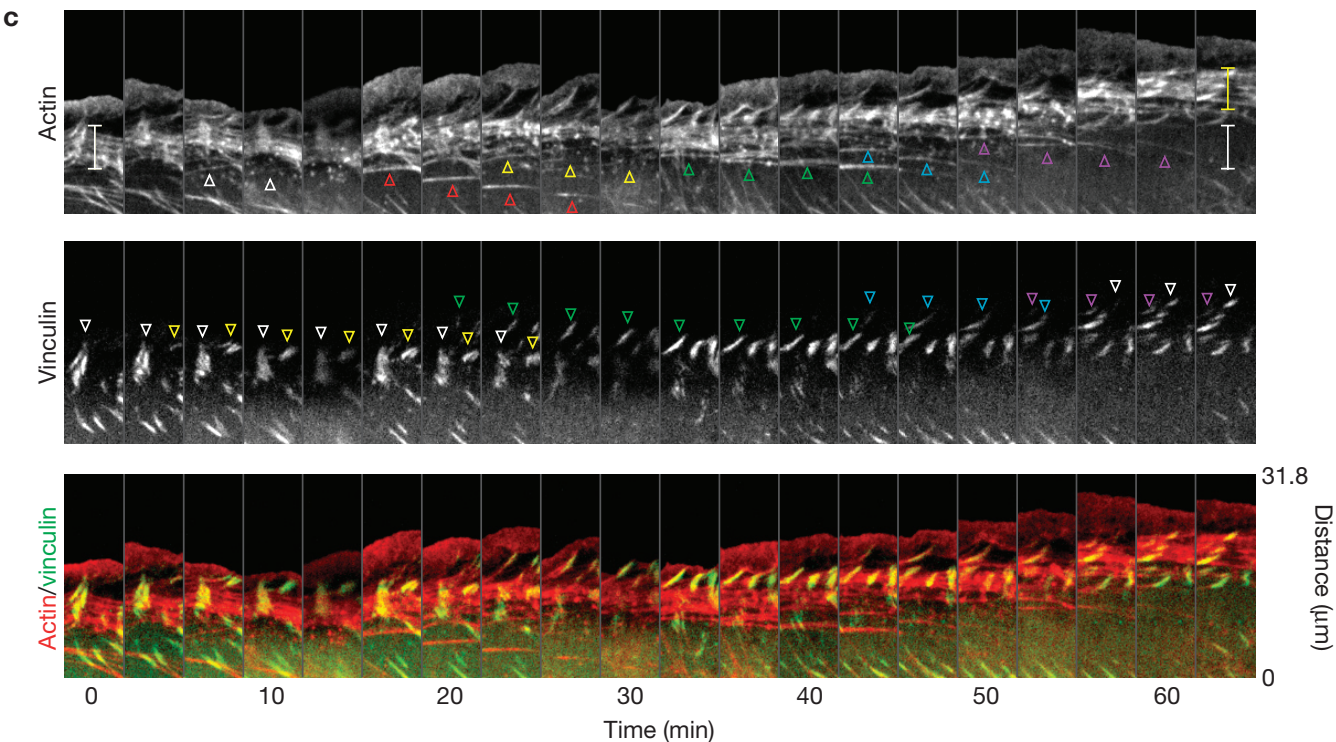

Figure 7 The advance of the lamella results from an actin-arc treadmill. (a) Actin-mGFP and (pseudo-coloured red) vinculin-mCherry (pseudo-coloured green) in a cell before and after net edge extension. Stars denote the lamellipodium and brackets denote the lamella. White arrowheads show focal adhesions at the boundary between the lamellipodium and lamella. Scale bar, $10 \mu \mathrm{m}$. (b) Edge-velocity map, edge-position map

dynamics from a cell that spontaneously started crawling reveals that the rate of advance of the transition between fast and slow actin-arc translocation can be changed within a single cell (Fig. 6g, arrows). Thus, the fundamental difference between cells crawling rapidly or slowly is not where or when actin arcs form, but other factors. One likely factor is the strength of coupling among actin arcs, focal adhesions and the substrate. This could determine the extent of focal-adhesion slippage backward and thus influence net forward movement.

\section{The advance of the lamella results from an actin-arc treadmill}

As a cell advances its edge, the lamellar stack of actin arcs maintains a relatively constant width (see Fig. 7a, brackets). Therefore, coordinated movement of the lamella itself must occur. To clarify how this works, we analysed the spatio-dynamics of actin arcs, focal adhesions and the structural features of the leading edge in a crawling cell. Analysis of and kymograph showing dynamics and advance of the leading edge. Dotted yellow lines denote advance of the lamella. (c) Actin montage of area outlined in a shows actin arcs are removed from the back of the lamella (arrowheads in actin montage) and new-focal-adhesion assembly (arrowheads in vinculin montage) leads to edge advance. Arrowhead colours denote distinct arcs or adhesions. The change in intensity in c from frame 5 to 6 is due to focusing.

both kymographs and time-lapse montages of actin arcs in a crawling cell reveals that the positional advance of new-actin-arc addition to the front of the lamella is balanced by the positional advance of the site of old-actin-arc removal at the rear of the lamella. (Fig. 7b, dashed line and Fig. $7 \mathrm{c}$, arrowheads in actin montage). This coordinated behaviour of arc addition in the front and removal in the rear of the arc stack may explain how cells avoid developing too many adhesive interactions with the substrate, which would impede movement during cell crawling.

\section{DISCUSSION}

In this study, we clarify the relationship between the lamellipodial and lamellar actin-filament modules at the cell's leading edge and relate this to cell crawling. We began by selectively photoconverting tdEos-labelled actin filaments in the lamellipodium and following their fate over time. The results were difficult to explain using previous models of 


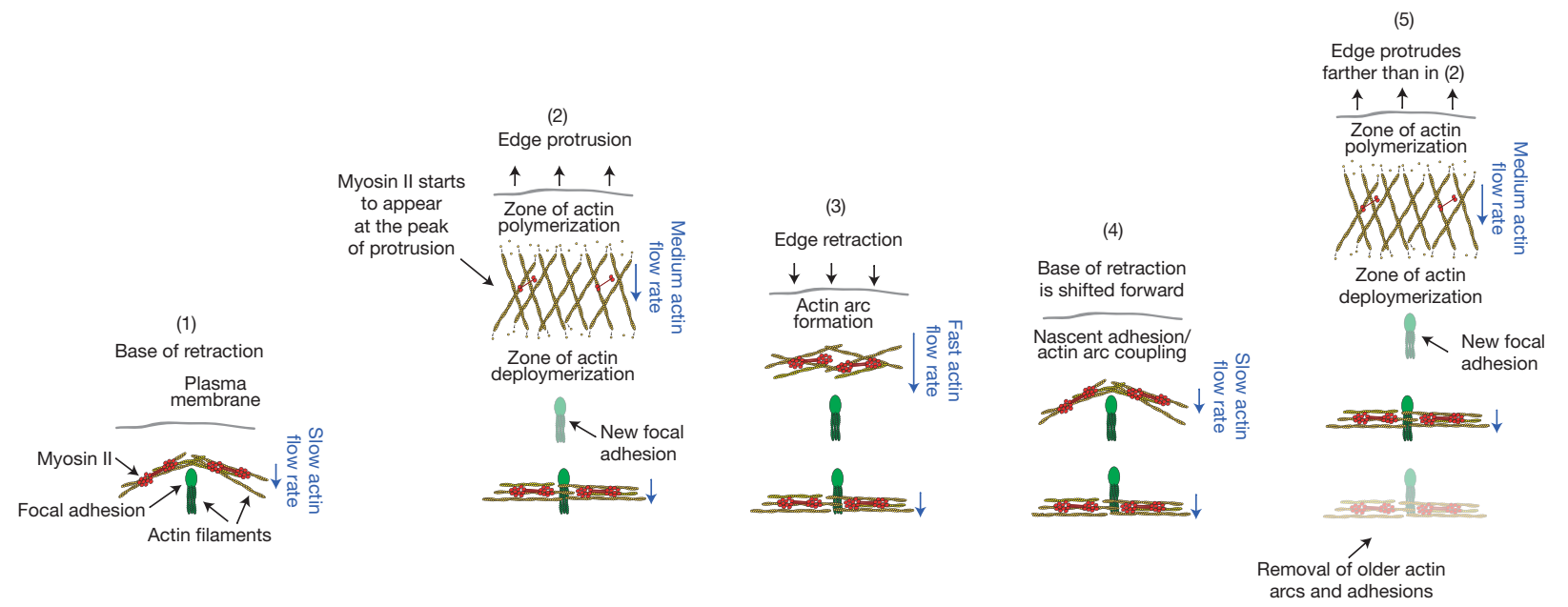

Figure 8 Model of the structural dynamics of the actin cytoskeleton underlying edge motion. Leading-edge advance is broken down into discrete steps. Step (1) shows the base of a previous retraction where a newly created actin arc is coupled to a focal adhesion. Hypothetically, the new lamellipodial protrusion could push off the arc to drive the membrane forward. During protrusion (2), actin-filament polymerization occurs behind the plasma membrane and depolymerization occurs a few micrometres away from the edge. Actin filaments treadmill through the lamellipodium during protrusion, and nascent adhesions form. At the peak of protrusion (2), myosin II filaments form in the lamellipodium and a local network contraction (similar to that proposed for keratocyte cell body translocation ${ }^{3}$ ) occurs that drives actin-arc formation and edge retraction (3). In cells that show net advance, the new actin arc slows at

lamellipodial and lamellar organization, which assumed the two actin modules are either dynamically distinct but overlapping networks ${ }^{11,15,17}$, or one continuous network ${ }^{5,16,30}$. Depending on whether the edge was protruding or retracting, the behaviour of the lamellipodial actin filaments differed (Fig. 2). During protrusion, labelled actin filaments quickly turned over with none reaching the lamella. During retraction, in contrast, converted filaments transferred to the lamella appearing in actin arcs.

Live-cell imaging using actin-mRFP helped explain the above results and supported an alternative model. The imaging revealed that actin arcs form at the cell edge soon after retraction is initiated. The arc then moves rearward, parallel to the edge, collecting with arcs in the lamella that are compiled as a stack. The presence of arcs as long-lived filaments in the lamellipodium can account for the differences in lifetimes of photoconverted tdEos-labelled actin filaments in the lamellipodium, and also explains previous FSM data showing long-lived speckles in the lamellipodium ${ }^{11}$. However, the data also raise questions, particularly because previous descriptions of arcs indicate that they form away from the edge in the lamella ${ }^{19,31}$, and in response to the presence of focal adhesions $s^{32}$. This led us to further analyse arc formation and dynamics. We found that the stack of arc bundles in the lamella derive from individual arcs formed in the lamellipodium (Fig. 2). Indeed, we observed that some individual arcs form in the lamellipodium before co-localization with focal adhesions (Fig. 3).

We next examined what drives the conversion of lamellipodial actin filaments into a rearward-moving actin arc. An obvious candidate was myosin II (refs 19,25). However, previous reports of myosin II distribution at the leading edge found myosin II restricted to the lamella ${ }^{11,13,26}$, suggesting that myosin II modulates

the nascent adhesion (4), most likely owing to strong coupling between the arc, adhesion and growth substrate. The base of the retraction in (4) is shifted forward when compared with (1). As a consequence, the start of the new protrusion in (5) is also shifted forward and the edge protrudes farther than in (2). In cells that do not show net advance, the actin arc and adhesion slip rearward during edge retraction. This indicates that there is still strong coupling between the actin arc and the adhesion, and also indicates a weak coupling between the adhesion and the growth substrate. Actin-arc addition to the front of the lamella is balanced by actin-arc removal at the back of the lamella (5). Lamellipodial and arc actin filaments are yellow. Focal adhesions and associated actin filaments are green. Myosin II filaments are red. Relative actin-rearward-flow rates are represented by blue arrows.

actin dynamics in the lamellipodium from a distance ${ }^{15}$. As these studies did not take into account potential differences in myosin II distribution when the leading edge was retracting or protruding, they might have missed a lamellipodial distribution of myosin II. Consistent with this possibility, high-resolution imaging of myosin IIA-GFP dynamics throughout the edge-protrusion/retraction cycle revealed myosin II filaments do form in the lamellipodium (Fig. 4). This occurs at the peak of the protrusion phase. The myosin filaments then move rearward with the newly forming actin arc during edge retraction.

The presence of myosin II in the lamellipodium raised the possibility that a local network contraction, similar to that proposed to drive the cell body in migrating fish keratocytes ${ }^{3}$, transforms the criss-crossed actin filaments in the lamellipodium into the bundled filaments that constitute the actin arc. Supporting this idea, no actin arcs form and the edge protrudes farther relative to that in control cells when myosin II activity is suppressed (Fig. 4). Forces exerted on the actin-filament cytoskeleton by focal adhesions may also play a role in arc formation ${ }^{32}$.

Given that lamellipodial actin converts into an arc parallel to the edge and subsequently moves rearward into the lamella, we next examined how this relates mechanistically to net edge extension and cell crawling. A clue came from our finding that the amplitude of the protrusion phase of the cell's oscillatory edge cycle remains constant and does not change even if the cell migrates forward. This means that net forward edge extension requires a process that shifts the base where the protrusion phase initiates. We found that the base where the protrusion phase initiates is the zone where the arc slows down on moving into the lamella (Fig. 6). This zone contains focal adhesions, which act as a brake on the rearward-moving arcs, slowing them down as they enter the 
lamella. This results in a single actin arc spanning many focal adhesions across the edge (Fig. 3). Stress fibres have recently been shown to mechanically link multiple large focal adhesions across the cell body in non-crawling cells ${ }^{33}$. Therefore, a linked actin-arc/focal-adhesion network at the leading edge could act during edge protrusion as a coherent stiff substrate for actin filaments in the lamellipodium to push back against to extend the plasma membrane.

On the basis of these results, we propose a model of leading-edge extension in which net forward edge movement occurs by nascent focal adhesions advancing the site at which new actin arcs slow down and thus where the next protrusion phase begins (Fig. 8). This would advance the base of the protrusion/retraction cycle in crawling cells. In non-crawling cells, the protrusion/retraction cycle of edge motion and its associated changes in actin dynamics are similar to crawling cells, but the actin arcs cause new adhesions to slip farther rearward during edge retraction. This results in little or no advance of the base of the protrusion/retraction cycle. The system thus behaves similarly to a mechanical ratchet, with the actin arc acting as the lever and focal adhesions acting as the teeth. The extent of slippage of focal adhesions backward, dictated by the strength of an adhesion with the substrate, determines how quickly the cell advances forward. Within this system, the lamella would stay roughly the same width but, on the whole, advance owing to new-actin-arc addition to the front of the lamella being balanced by removal of older actin arcs at the back of the lamella, as in a treadmill.

A cyclic myosin-II-based actin-arc formation and dissolution mechanism may also underlie the motile behaviour in a variety of cells because most cells exhibit oscillatory edge protrusion and retraction. Indeed, other motile cells have both a protrusion/retraction cycle and actin arcs. The list includes, but is not limited to, fish keratocytes, neuronal growth cones and mouse melanoma cells ${ }^{3,25,34}$ (Supplementary Fig. S4 and Movie S4). Our proposal of leading-edge motion involving myosin-II-based actin-arc formation and dissolution thus provides a predictive model for cell crawling testable in a range of cell types.

\section{METHODS}

Methods and any associated references are available in the online version of the paper at http://www.nature.com/naturecellbiology/

Note: Supplementary Information is available on the Nature Cell Biology website

\section{ACKNOWLEDGEMENTS}

We thank the members of the Lippincott-Schwartz Laboratory for helpful comments and suggestions. D.T.B. was supported by a Pharmacology Research Associate Fellowship from NIGMS, NIH during the course of these studies.

\section{AUTHOR CONTRIBUTIONS}

D.T.B., S.M. and J.L-S. designed experiments and wrote the paper. D.T.B. carried out the experiments. D.T.B., S.M. and P.S. analysed the data. M.W.D. contributed new fluorescence probes. R.S. and B.K. contributed expertise in electron microscopy.

\section{COMPETING FINANCIAL INTERESTS}

The authors declare no competing financial interests.

Published online at http://www.nature.com/naturecellbiology

Reprints and permissions information is available online at http://npg.nature.com/ reprintsandpermissions/

1. Rafelski, S. M. \& Theriot, J. A. Crawling toward a unified model of cell mobility: spatial and temporal regulation of actin dynamics. Annu. Rev. Biochem. 73, 209-239 (2004).

2. Wang, Y. L. Exchange of actin subunits at the leading edge of living fibroblasts: possible role of treadmilling. J. Cell Biol. 101, 597-602 (1985).
3. Svitkina, T. M., Verkhovsky, A. B., McQuade, K. M. \& Borisy, G. G. Analysis of the actin-myosin II system in fish epidermal keratocytes: mechanism of cell body translocation. J. Cell Biol. 139, 397-415 (1997)

4. Svitkina, T. Electron microscopic analysis of the leading edge in migrating cells. Methods Cell Biol. 79, 295-319 (2007).

5. Koestler, S. A., Auinger, S., Vinzenz, M., Rottner, K. \& Small, J. V. Differentially oriented populations of actin filaments generated in lamellipodia collaborate in pushing and pausing at the cell front. Nat. Cell Biol. 10, 306-313 (2008).

6. Urban, E., Jacob, S., Nemethova, M., Resch, G. P. \& Small, J. V. Electron tomography reveals unbranched networks of actin filaments in lamellipodia. Nat. Cell Biol. 12, 429-435 (2010).

7. Heath, J. P. Arcs: curved microfilament bundles beneath the dorsal surface of the leading lamellae of moving chick embryo fibroblasts. Cell Biol. Int. Rep. 5, 975-980 (1981).

8. Gupton, S. L. \& Waterman-Storer, C. M. Spatiotemporal feedback between actomyosin and focal-adhesion systems optimizes rapid cell migration. Cell 125, 1361-1374 (2006).

9. Forscher, P. \& Smith, S. J. Actions of cytochalasins on the organization of actin filaments and microtubules in a neuronal growth cone. J. Cell Biol. 107, 1505-1516 (1988).

10. Pollard, T. D. \& Borisy, G. G. Cellular motility driven by assembly and disassembly of actin filaments. Cell 112, 453-465 (2003).

11. Ponti, A., Machacek, M., Gupton, S. L., Waterman-Storer, C. M. \& Danuser, G. Two distinct actin networks drive the protrusion of migrating cells. Science 305, 1782-1786 (2004).

12. Hotulainen, P. \& Lappalainen, P. Stress fibers are generated by two distinct actin assembly mechanisms in motile cells. J. Cell Biol. 173, 383-394 (2006).

13. Giannone, G. et al. Periodic lamellipodial contractions correlate with rearward actin waves. Cell 116, 431-443 (2004).

14. Danuser, G. Coupling the dynamics of two actin networks-new views on the mechanics of cell protrusion. Biochem. Soc. Trans. 33, 1250-1253 (2005).

15. Giannone, G. et al. Lamellipodial actin mechanically links myosin activity with adhesion-site formation. Cell 128, 561-575 (2007).

16. Vallotton, P. \& Small, J. V. Shifting views on the leading role of the lamellipodium in cell migration: speckle tracking revisited. J. Cell Sci. 122, 1955-1958 (2009).

17. Danuser, G. Testing the lamella hypothesis: the next steps on the agenda. J. Cell Sci. 122, 1959-1962 (2009).

18. Mogilner, A. \& Keren, K. The shape of motile cells. Curr. Biol. 19, R762-R771 (2009).

19. Machacek, M. \& Danuser, G. Morphodynamic profiling of protrusion phenotypes. Biophys. J. 90, 1439-1452 (2006).

20. Small, J. V. et al. Unravelling the structure of the lamellipodium. J. Microsc. 231, 479-485 (2008).

21. Svitkina, T. M., Verkhovsky, A. B. \& Borisy, G. G. Improved procedures for electron microscopic visualization of the cytoskeleton of cultured cells. J. Struct. Biol. 115, 290-303 (1995).

22. Waterman-Storer, C. M., Desai, A., Bulinski, J. C. \& Salmon, E. D. Fluorescent speckle microscopy, a method to visualize the dynamics of protein assemblies in living cells. Curr. Biol. 8, 1227-1230 (1998).

23. Hu, K., Ji, L., Applegate, K. T., Danuser, G. \& Waterman-Storer, C. M. Differential transmission of actin motion within focal adhesions. Science $\mathbf{3 1 5}$, 111-115 (2007).

24. Gardel, M. L. et al. Traction stress in focal adhesions correlates biphasically with actin retrograde flow speed. J. Cell Biol. 183, 999-1005 (2008).

25. Medeiros, N. A., Burnette, D. T. \& Forscher, P. Myosin II functions in actin-bundle turnover in neuronal growth cones. Nat. Cell Biol. 8, 215-226 (2006).

26. Choi, C. K. et al. Actin and $\alpha$-actinin orchestrate the assembly and maturation of nascent adhesions in a myosin II motor-independent manner. Nat. Cell Biol. 10, 1039-1050 (2008).

27. Webb, D. J., Parsons, J. T. \& Horwitz, A. F. Adhesion assembly, disassembly and turnover in migrating cells-over and over and over again. Nat. Cell Biol. 4, E97-E100 (2002).

28. Alexandrova, A. Y. et al. Comparative dynamics of retrograde actin flow and focal adhesions: formation of nascent adhesions triggers transition from fast to slow flow. PLoS One 3, e3234 (2008).

29. Aratyn-Schaus, Y. \& Gardel, M. L. Transient frictional slip between integrin and the ECM in focal adhesions under myosin II tension. Curr. Biol. 20, 1145-1153 (2010).

30. Wilson, C. A. et al. Myosin II contributes to cell-scale actin network treadmilling through network disassembly. Nature 465, 373-377 (2010).

31. Zhang, X. F., Schaefer, A. W., Burnette, D. T., Schoonderwoert, V. T. \& Forscher, P. Rho-dependent contractile responses in the neuronal growth cone are independent of classical peripheral retrograde actin flow. Neuron 40, 931-944 (2003).

32. Shemesh, T., Verkhovsky, A. B., Svitkina, T. M., Bershadsky, A. D. \& Kozlov, M. M. Role of focal adhesions and mechanical stresses in the formation and progression of the lamellipodium-lamellum interface [corrected]. Biophys. J. 97, 1254-1264 (2009).

33. Rossier, O. M. et al. Force generated by actomyosin contraction builds bridges between adhesive contacts. EMBO J. 29, 1055-1068 (2010).

34. Mongiu, A. K., Weitzke, E. L., Chaga, O. Y. \& Borisy, G. G. Kinetic-structural analysis of neuronal growth cone veil motility. J. Cell Sci. 120, 1113-1125 (2007). 


\section{METHODS}

Cell culture and chemicals. PtK1 cells were purchased from ATCC and cultured in DMEM F12 (Invitrogen) as previously described ${ }^{8,35}$. Cells were plated on coverslips coated with $10 \mu \mathrm{g} \mathrm{ml} l^{-1}$ fibronectin for $2 \mathrm{~h}$ at $37^{\circ} \mathrm{C}$ and cultured overnight. Cells were transfected with DNA plasmids with fugene (Roche) following the manufacturer's recommendations, and allowed to express for $12 \mathrm{~h}$. Cells were then imaged in $\mathrm{CO}_{2}$-independent media (Invitrogen) at $37^{\circ} \mathrm{C}$. Blebbistatin, taxol, phalloidin and fibronectin were from Sigma.

Actin-speckle tracking. Time-lapse images of actin speckles were acquired with both wide-field epifluorescence and spinning disc microscopy at intervals of $5 \mathrm{~s}$ with an integration time of 300-600 ms with an Ultraview spinning disc confocal microscope (Perkin Elmer) attached to an Olympus IX71 microscope (Olympus) or a Marianas spinning disc microscope (Intelligent Imaging Innovations) attached to a Zeiss Observer.Z1 microscope (Carl Zeiss). Actin speckles were generated by selective conversion of a subpopulation of actin-tdEos molecules by a 100-400 ms exposure with $405 \mathrm{~nm}$ laser light, through the spinning disc or by use of a Mosaic Digital Illumination System (Photonic Instruments). An adaptive multi-frame correlation approach, as previously described ${ }^{36,37}$, was used to track actin-tdEos speckles. Our effective temporal resolution, obtained by averaging three frames acquired at 5-s intervals, was $15 \mathrm{~s}$, which provided sufficient resolution to determine whether there were changes in actin-flow rates over the cycle of protrusion and retraction underlying edge motion, which is of the order of minutes. A small amount of actin-tdEos was converted to the red channel in the cell body every 40-50 frames. These monomers then slowly incorporated into the actin network at the leading edge, thus replenishing the pull of actin speckles lost to photobleaching.

Photoconversion of actin-tdEos in specific cellular regions. Actin-tdEos photoconversion experiments presented in Fig. 2 were carried out on a Marianas spinning disc confocal microscope equipped with a Mosaic Digital Illumination System. The laser power entering the Mosaic was $9 \mathrm{~mW}$. Image acquisition and photoconversion of actin-tdEos molecules (including region selection and 405 laser exposure control) were carried out using Slidebook 5.0 software. All other experiments were carried out with actin fused to monomeric fluorescent proteins The incorporation of actin constructs into filaments in every observable actin-based structure was confirmed with live-cell extraction followed by a comparison with fluorescent phalloidin labelling.

Electron microscopy. Electron microscopy of rotary-shadowed cells was carried out as previously described ${ }^{38}$. Briefly, cells were extracted with $1 \%$ Triton X-100 in cytoskeleton stabilization buffer (100 mM PIPES, at $\mathrm{pH} 6.9,4 \%$ polyethylene glycol, $10 \mu \mathrm{M}$ phalloidin, $10 \mu \mathrm{M}$ Taxol, $5 \mathrm{mM}$ EGTA and $5 \mathrm{mM} \mathrm{MgCl}_{2}$ ) for $5 \mathrm{~min}$ at room temperature. Cells were then washed with wash buffer (100 mM PIPES, at pH 6.9 $10 \mu \mathrm{M}$ phalloidin, $10 \mu \mathrm{M}$ Taxol, $5 \mathrm{mM}$ EGTA and $5 \mathrm{mM} \mathrm{MgCl}_{2}$ ) for 2 min and fixed with $2 \%$ glutaraldehyde for $20 \mathrm{~min}$. Fixation with $0.1 \%$ tannic acid $(20 \mathrm{~min})$ was followed by treatment with $0.2 \%$ uranyl acid $(20 \mathrm{~min})$. Samples were dehydrated with increasing concentrations of ethanol $(10 \%, 30 \%, 50 \%, 70 \%, 90 \%, 100 \%, 100 \%$, $100 \%$ and $100 \%$ dried with molecular sieves purchased from Sigma) for 5 min each. Cells were critical-point dried and rotary shadowed with platinum/carbon. All steps were done at room temperature. Images were acquired at $80 \mathrm{kV}$.

Analysis. Time-lapse images of actin-RFP were acquired at intervals of 10-20 s with an Ultraview spinning disc or a Marinas spinning disc microscope. Leading-edge protrusion and retraction velocities were measured as previously described ${ }^{11,19}$ The software package used defines a spline representation of the leading edge and calculates edge displacements between frames. The spacing between displacement measurements along the edge was set at 2 pixels. Custom software was developed using Matlab (The Mathworks) to convert displacement measurements to velocities in nanometres per second. The edge was divided into consecutive 172-nm-wide positions and velocities for each position over time were binned. For each cell, two matrices were constructed that contained the edge displacements and velocities, respectively, along different spatial positions (each matrix had $N$ columns corresponding to $N$ spatial positions interrogated). In the displacement matrix, each column represented a separate spatial position and contained the edge-displacement values of that position at different time points. Similarly, each column in the velocity matrix contained the velocity values corresponding to the evolution of velocity over time at that particular spatial position.

Measurement of protrusion/retraction period. The primary frequency of velocity fluctuation at each spatial position (bin) was identified by Fourier transforming the velocity values drawn from the corresponding column of the velocity matrix, and then the time period was subsequently calculated using this primary frequency. Finally, the characteristic frequency and time period of protrusion/retraction of the cell were calculated by averaging the primary frequencies and time periods, respectively, of all the spatial positions.

Protrusion-amplitude measurements. For each cell, the time points for the start and end of each protrusion were initially calculated using the velocity matrix (constructed as described in the previous section). The change in sign of velocity values was used to identify the start and end of protrusions, with the velocity values changing from negative to positive at the start of a protrusion (and end of preceding retraction), and, changing from positive to negative at the end of the protrusion (and start of the next retraction). To discard the extraneous cycles arising from local fluctuations of velocity values during the transition from protrusion to retraction (and vice versa), the cycles of protrusion/retraction with time period less than half of the time period (for that particular cell) identified by Fourier transformation (described in the previous section) were excluded.

Once the time points of start and end of protrusions were identified, the amplitude of each individual protrusion was calculated from the differences in displacements of each spatial position between the end and start of the protrusion. The retraction amplitudes were similarly calculated by determining the difference between the end and start of the retractions (represented by the start of protrusion and the end of the previous protrusion). The average protrusion and retraction amplitudes of edge motion for each cell were obtained by averaging the corresponding values for all of the spatial positions.

Measurement of edge growth. Finally, the net edge growth for each cell was calculated from the differences in displacements of each spatial position between the position of the base of the first protrusion and the position of the base of the last protrusion. The edge growth rate in micrometres per minute could subsequently be calculated by dividing the net edge growth by the time interval between the two spatial positions.

Focal-adhesion movement. Centroids of focal adhesions labelled with either zyxin-mCherry or vinculin-mCherry were tracked using the manual-tracking protocol included in Slidebook. Next, a custom-written Matlab code was used to measure the displacement of the newly formed focal adhesions over two protrusion/retraction cycles from their first point of appearance. Briefly, the protrusion or retraction cycle during which the focal adhesions first appeared was identified using the start/end points of the protrusion/retraction cycles obtained as described previously, and the position of the closest pre-existing focal adhesion for every new focal adhesion was determined. Finally, the displacement and the net displacement (displacement from the closest pre-existing focal adhesion) of each newly formed focal adhesion were calculated by tracking their spatial evolution during two consecutive protrusion/retraction cycles following their appearance.

35. Wittmann, T., Bokoch, G. M. \& Waterman-Storer, C. M. Regulation of leading edge microtubule and actin dynamics downstream of Rac1. J. Cell Biol. 161, 845-851 (2003).

36. Ji, L. \& Danuser, G. Tracking quasi-stationary flow of weak fluorescent signals by adaptive multi-frame correlation. J. Microsc. 220, 150-167 (2005).

37. Burnette, D. T. et al. Myosin II activity facilitates microtubule bundling in the neuronal growth cone neck. Dev. Cell 15, 163-169 (2008).

38. Burnette, D. T., Schaefer, A. W., Ji, L., Danuser, G. \& Forscher, P. Filopodial actin bundles are not necessary for microtubule advance into the peripheral domain of Aplysia neuronal growth cones. Nat. Cell Biol. 9, 1360-1369 (2007). 


\section{cell biology}

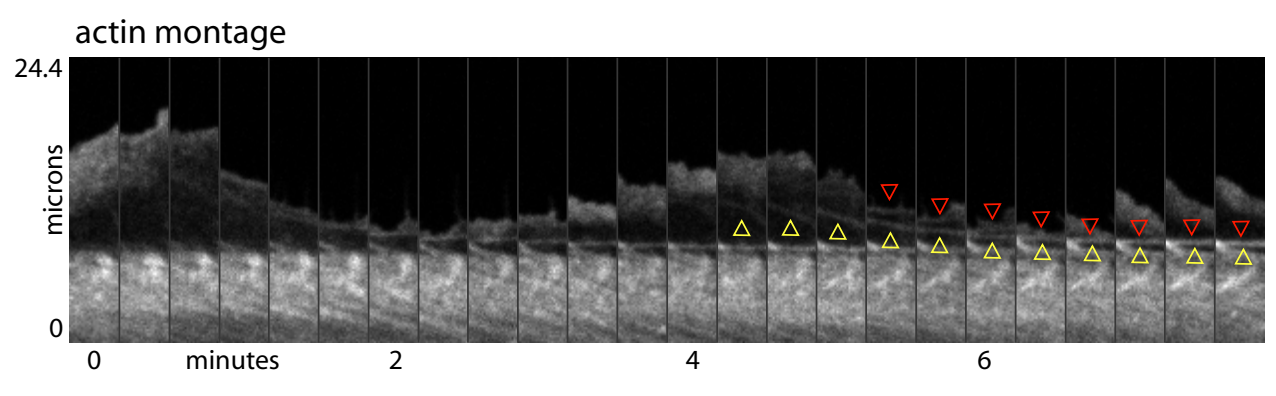

Figure S1 Time montage showing a secondary actin bundle left behind by the protruding lamellipodium (yellow arrowhead). This bundle merges with the newly formed actin arc (red arrowhead) during edge retraction. 
a
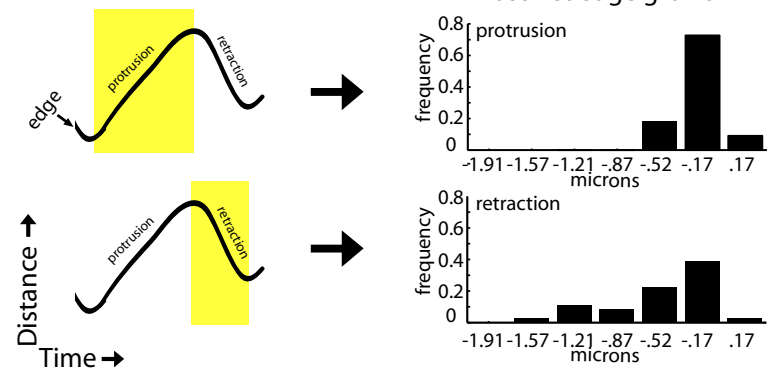

C adhesion displacemen slow net edge growth
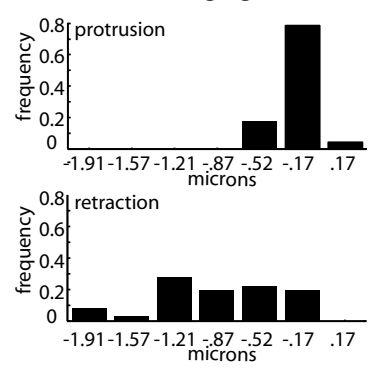

Figure S2 Focal adhesion slippage occurs during edge retraction. a) Schematic showing what time segmentation of focal adhesion movement quantified in b) and c). b) Total focal adhesion translocation during the protrusion phase of edge motion. c) Total focal adhesion translocation during the retraction phase of edge motion. 
a
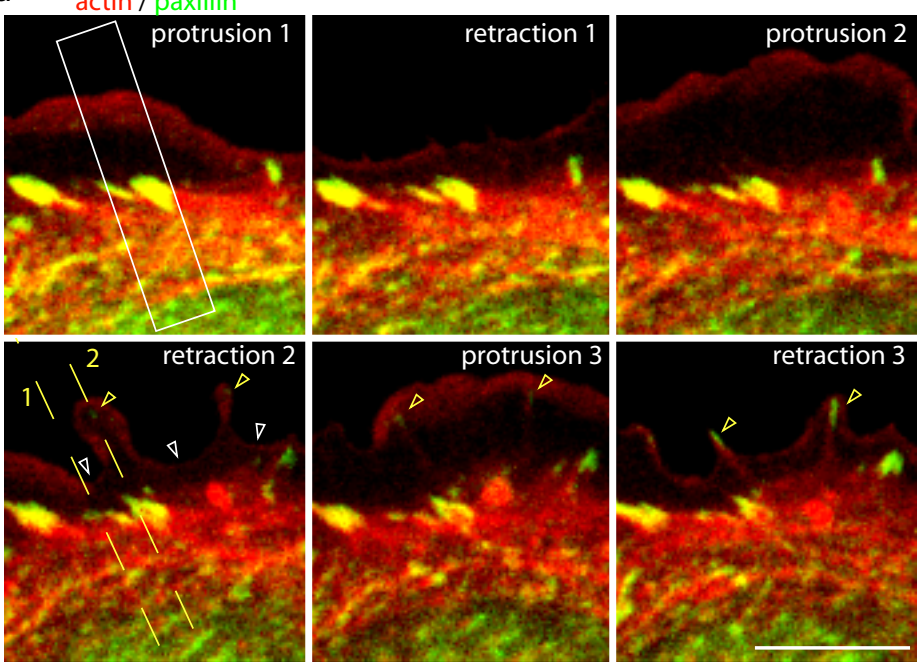

d montage- box in (a)

$\nabla$ nascent adhesion formation
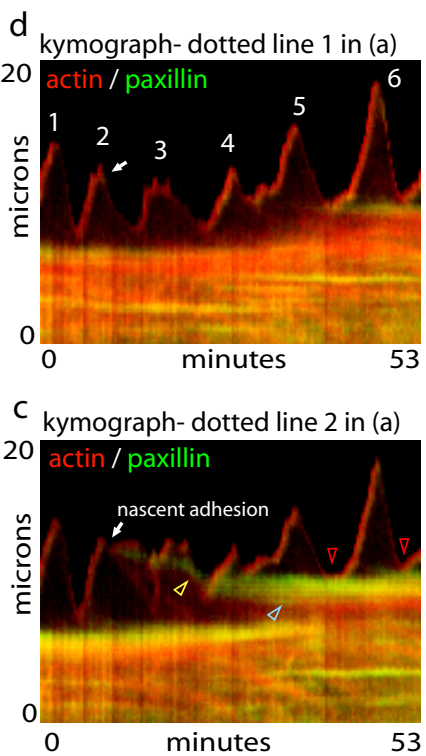
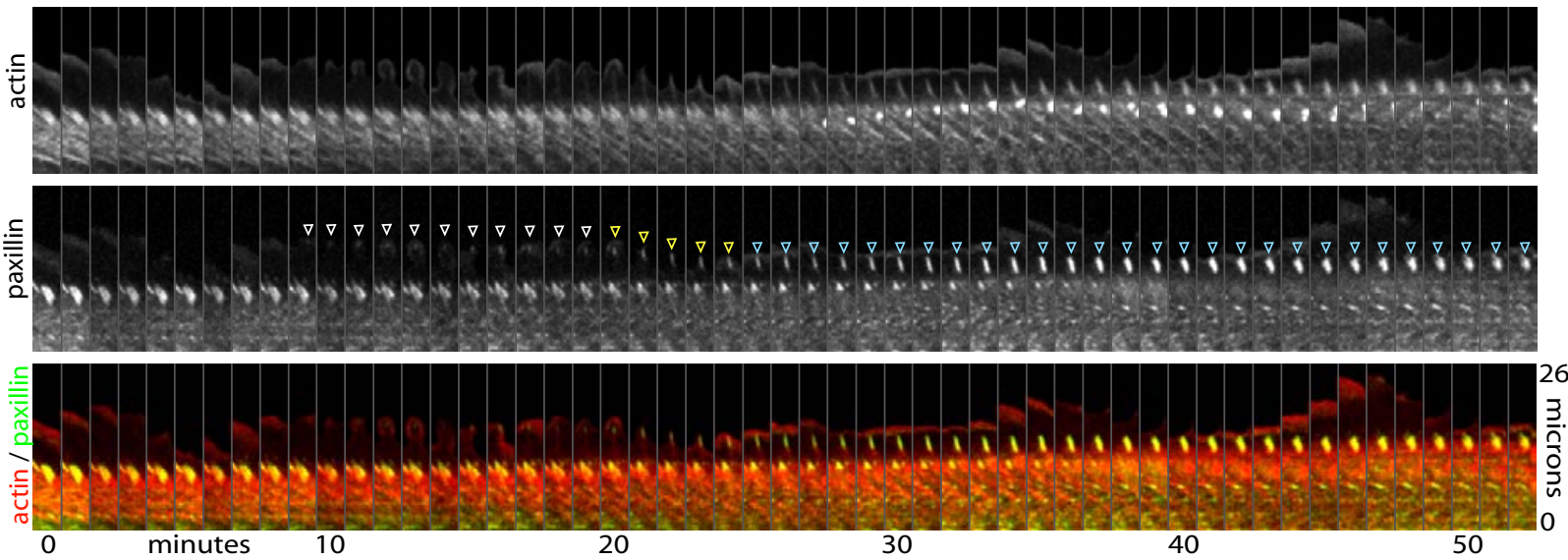

Figure S3 Actin arc coupling to nascent adhesions advances the edge's protrusion retraction cycle. a) Time montage of paxllin-mCherry and actinmGFP over three protrusion retraction cycles. Yellow arrowheads denote nascent adhesions and white arrowheads denote actin arc retraction between nascent adhesions. b-c) Paxillin (green) and actin (red) kymographs from line 1 (b) and line 2 (c) in (A). Line 1 is adjacent to the nascent adhesion formation and line 2 is a region directly on the adhesion. The number of protrusion/retraction cycles are labeled in (b). Arrowheads denote nascent adhesion formation. (c) Yellow arrowhead denotes rearward movement of the nascent adhesion and blue arrowhead denotes maturation. Red arrowheads denote the advance of the location of the base of the protrusion/retraction cycle after adhesion maturation. d) Time-montage of the box in (a). White arrowheads denote the appearance, yellow arrowheads denote the rearward movement, and blue arrows denote the maturation of adhesion. 
a fish keratocyte

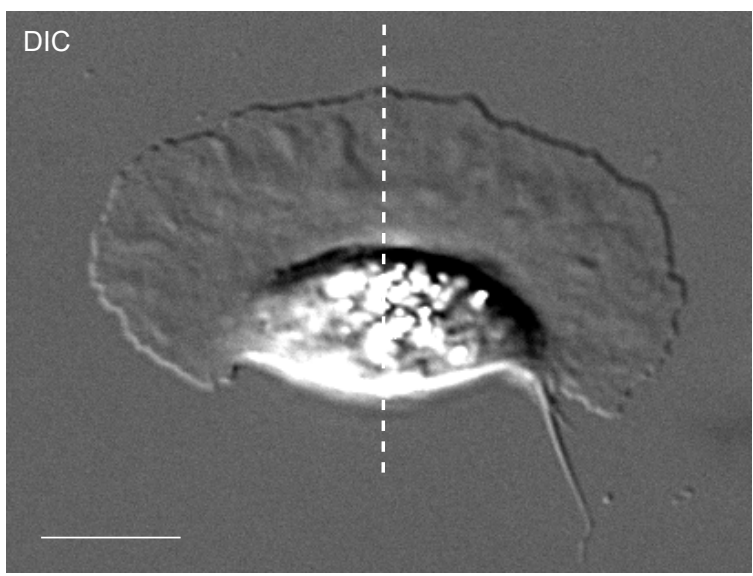

b

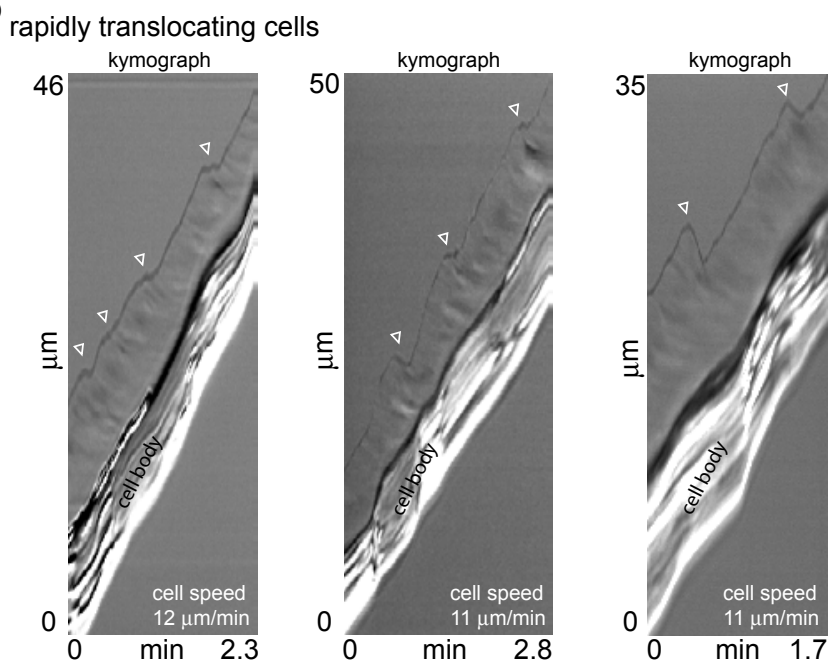

C slowly translocating cells

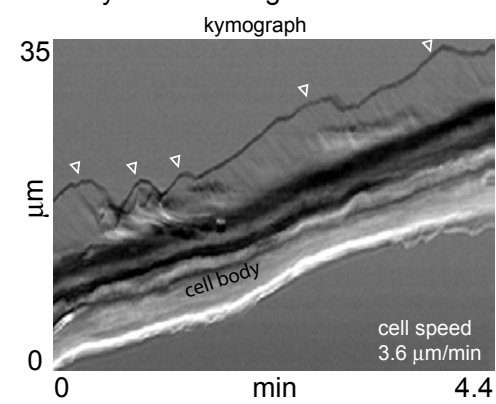

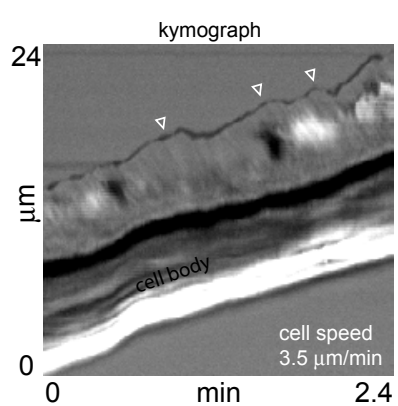

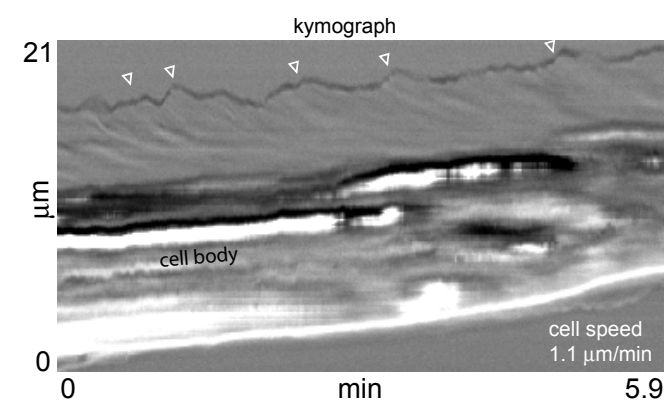

Figure S4 Oscillatory edge motion at the leading edge of migrating fish keratocyes. Fish keratocytes have long been a favored model for cell migration because of their extremely fast migration rates. Although, electron microscopy studies have shown keratocytes have prominent myosin IIladen actin arcs, it has been assumed that their edge only protrudes, and, thus, does not display oscillatory motion 1,2 . We found that the published data was not sufficient to distinguish whether or not fish keratocytes had oscillatory edge motion. Therefore, we cultured keratocytes as previously described ${ }^{3}$ from the scales of Poecilia latipinna. We then acquired differential interference contrast time-lapse recordings of single crawling cells with images acquired every second. a) shows one frame of a time-lapse DIC recording of one of these crawling cells (dotted line shows orientation of kymographs). b-c) Kymographs are presented for three cells that are moving quickly (b) and slowly (c) relative to each other. All cells analyzed show edge oscillations (arrowheads). These oscillations do not always, but indeed can, result from an edge retraction, but, instead, a slowing of the edge protrusion. Cell body and cell velocity are indicated in the kymograph for each cell. Actin arc formation has been shown to be cyclical and myosin II-dependent in the neuronal growth cone with little, if any edge oscillations. It is important to notes that these studies were done using growth cones on polyD-lysine coated coverslips on which growth cones do not extend. In contrast, neuronal growth cones plated on permissive growth substrates do show a robust edge oscillatory cycle identical to the motion presented here. The similarity in actin filament organization between the keratocyte ${ }^{1}$, neuronal growth cone 4,5 , and PtK 1 cells (Fig. 1 ) gives further support that these cells could use the same mechanism for motility. 


\section{Supplemental Movie Legends}

Movie S1 Raw time-lapse recording of actin-tdEOS molecules at the leading edge. Epi-fluorescence. Images acquired every 5 seconds. Movie height: 33 mm. Movie length: $15.3 \mathrm{~min}$.

Movie S2 Time-lapse recording of actin-RFP expressed in a PtK1 cell. Spinning disk confocal. Images acquired every 20 seconds. Movie height: $39 \mu \mathrm{m}$. Movie length: $60 \mathrm{~min}$.

Movie S3 Time-lapse recording of actin-RFP (red) and myosin IIA-GFP (green) at the leading edge. Myosin IIA-GFP channel intensity was stretched to reveal the small amount of myosin in the lamellipodium. Spinning disk confocal. Images acquired every 10 seconds. Movie height: $31 \mu \mathrm{m}$. Movie length: 25.8 min.

Movie S4 Time-lapse recording of a mouse melanoma cell (B16-F1) expressing actin-mRFP. Note the cycle of actin arc assembly and disassembly underlying edge motion. It has been shown that actin filaments from the filopodial actin bundles (see prominent actin bundles a the leading edge) can be added to the actin arc bundles ${ }^{6}$. How this modification changes edge growth will be an interesting direction of future investigation. We focused our current study on actin arcs in cells that lack filopodial actin bundles for several reasons. First, actin arc assembly occurs in cells lacking filopodia ${ }^{1}$. Secondly, it has been shown that actin arc formation is unimpeded by filopodial actin bundle removal ${ }^{4}$. Taken together, this would indicate that the basal mechanism of actin arc format ion is independent of filopodia. B16-F1 cells were cultured as previously describe ${ }^{6}$. Images were acquired every 5 seconds. Movie height: $61 \mu \mathrm{m}$. Movie length: 14 min.

\section{References for Supplemental Information}

1. Svitkina, T. M., Verkhovsky, A. B., McQuade, K. M. \& Borisy, G. G. Analysis of the actin-myosin II system in fish epidermal keratocytes: mechanism of cell body translocation. J Cell Biol 139, 397-415 (1997).

2. Machacek, M. \& Danuser, G. Morphodynamic profiling of protrusion phenotypes. Biophys J 90, 1439-52 (2006).

3. Theriot, J. A. \& Mitchison, T. J. Actin microfilament dynamics in locomoting cells. Nature 352, 126-31 (1991).

4. Burnette, D. T., Schaefer, A. W., Ji, L., Danuser, G. \& Forscher, P. Filopodial actin bundles are not necessary for microtubule advance into the peripheral domain of Aplysia neuronal growth cones. Nat Cell Biol 9, 1360-9 (2007).

5. Mongiu, A. K., Weitzke, E. L., Chaga, O. Y. \& Borisy, G. G. Kinetic-structural analysis of neuronal growth cone veil motility. J Cell Sci 120, 111325 (2007).

6. Nemethova, M., Auinger, S. \& Small, J. V. Building the actin cytoskeleton: filopodia contribute to the construction of contractile bundles in the lamella. J Cell Biol 180, 1233-44 (2008). 